Solvation Induction of Free Energy Barriers of Decarboxylation Reactions in Aqueous Solution from Dual-level QM/MM Simulations

Shaoyuan Zhou, ${ }^{1,2}$ Yingjie Wang, ${ }^{2}$ and Jiali Gao ${ }^{2,3,4}$

1. Institute of Theoretical Chemistry, Jilin University, Changchun, 130023, China

2. Institute of Systems and Physical Biology, Shenzhen Bay Laboratory, Shenzhen 518055, China

3. Beijing University Shenzhen Graduate School, Shenzhen 518055, China

4. Department of Chemistry and Supercomputing Institute, University of Minnesota, Minneapolis, MN 55455, USA

\title{
AUTHOR INFORMATION
}

Email: S.Z., shaoyuan303@icloud.com;

Y.W., wangyj@szbl.ac.cn;

Corresponding Authors

J.G., gao@jialigao.org; 


\section{Supporting Information}

\section{Contents}

Table S1. Decarboxylation reactions of 11 anions in water and details of QM/MM simulation models..3

Table S2. Hydrogen bond distances and the interaction energies of M06-2X and QM/MM results for the 34 bimolecular complexes in Figure S2.

Table S3. Computational and experimental gas-phase energies along the decarboxylation reaction path.

Table S4 Optimized values of the Lennard-Jones (LJ) rmin and $\varepsilon$ parameter for the description of the vDW interaction between representative atom pairs in Figure 1.

Table S5. Estimation of free energies of reaction for the decarboxylation of N-methylorotate and nitroacetate in water based on experimental acidity constants $\mathrm{pKa}$ and computed free energy difference of the corresponding decarboxylation of the neutral acids.

Table S6. The decomposition of the QM/MM interaction energy of reactant, transition and product state.

Figure S1. Some geometry parameters of the optimized equilibrium structures obtained at high-level M06-2X/aug-cc-pVTZ and low-level semiempirical AM1 method.

Figure S2. Bimolecular complexes along with computed binding energies and optimized hydrogen bond distance for carboxylate anions (A) and carbanions (B) with a water molecule at different interaction sites.

Figure S3. Energy terms in Eq. 10 and variations in solvation free energy along the reaction path and the final corrected PMFs of along the decarboxylation reaction coordinates.

Figure S4. Correlation between calculated $\Delta E_{X s}^{\text {elec }}$ and $\Delta E_{X s}^{\text {pol }}$ .13

Figure S5-1-6. Radial distribution functions (RDFs) for several hydrogen bond interaction pairs at different states. 
Table S1. Decarboxylation reactions of 11 anions in water and details of QM/MM simulation models.

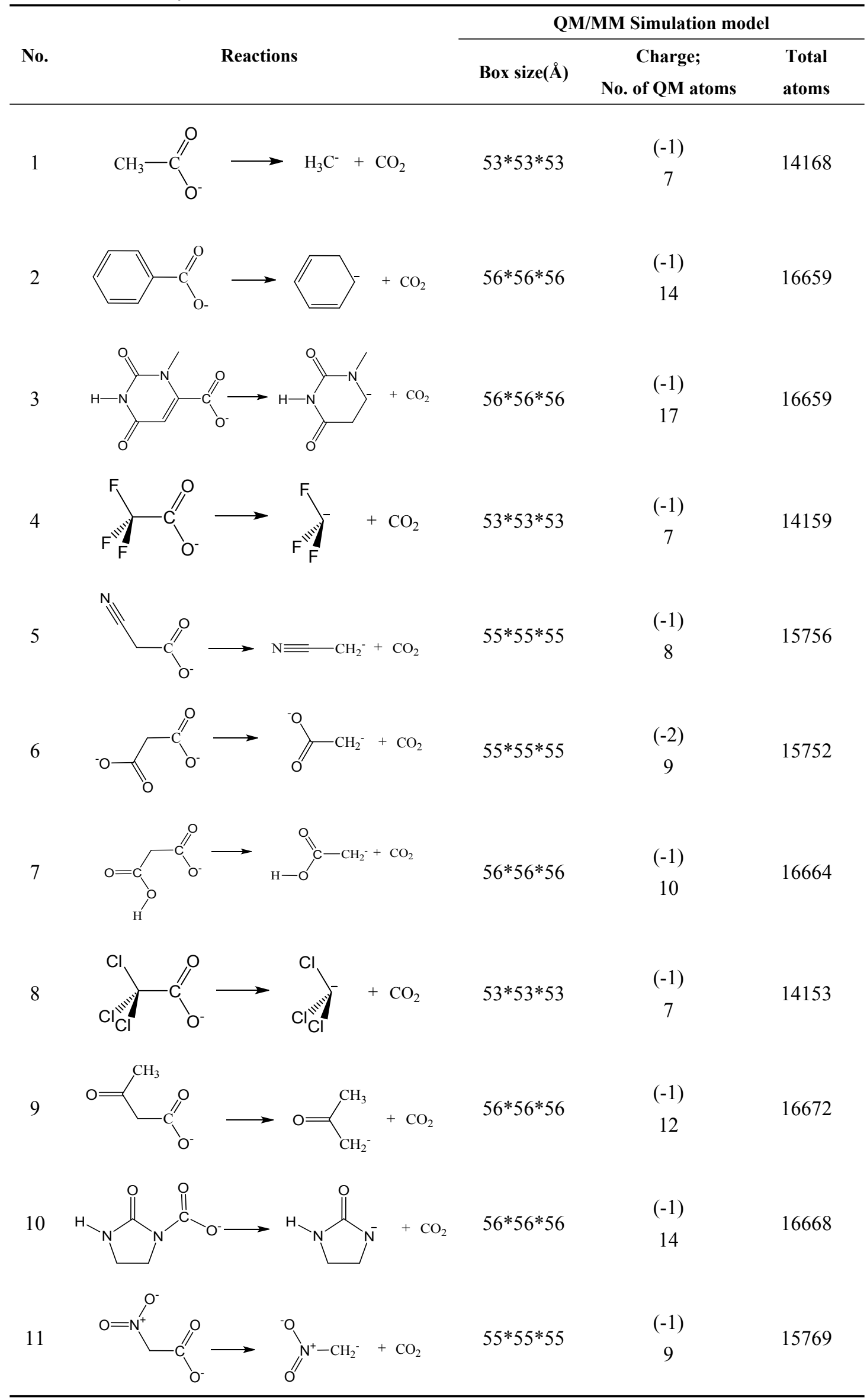


Table S2. Hydrogen bond distances and the interaction energies of M06-2X and QM/MM results for the 34 bimolecular complexes in Figure S2.

\begin{tabular}{|c|c|c|c|c|}
\hline Index & $\mathbf{R}(\AA ̊)-\mathbf{Q M}$ & $\mathbf{R}(\AA)-\mathbf{Q M} / \mathbf{M M}$ & $\Delta \mathrm{E}(\mathrm{kcal} / \mathrm{mol})-\mathrm{QM}$ & $\Delta \mathrm{E}(\mathrm{kcal} / \mathrm{mol})-\mathrm{QM} / \mathrm{MM}$ \\
\hline 1 & 3.13 & 3.17 & -19.5 & -19.8 \\
\hline 2 & 3.14 & 3.17 & -17.9 & -18.6 \\
\hline 3 & 3.16 & 3.17 & -15.2 & -15.2 \\
\hline 4 & 1.83 & 1.85 & -8.7 & -9.0 \\
\hline 5 & 1.79 & 1.86 & -9.6 & -9.2 \\
\hline 6 & 1.97 & 1.98 & -1.9 & -2.0 \\
\hline 7 & 3.14 & 3.16 & -16.2 & -15.3 \\
\hline 8 & 1.97 & 1.95 & -6.1 & -7.3 \\
\hline 9 & 1.97 & 1.96 & -5.6 & -6.8 \\
\hline 10 & 3.14 & 3.17 & -16.7 & -17.9 \\
\hline 11 & 1.96 & 1.96 & -8.8 & -8.4 \\
\hline 12 & 3.10 & 3.18 & -25.6 & -24.3 \\
\hline 13 & 3.10 & 3.17 & -25.2 & -24.3 \\
\hline 14 & 3.14 & 3.16 & -17.3 & -17.7 \\
\hline 15 & 1.88 & 1.90 & -8.1 & -8.3 \\
\hline 16 & 1.83 & 1.83 & -9.5 & -9.8 \\
\hline 17 & 2.02 & 2.13 & -8.6 & -8.3 \\
\hline 18 & 1.94 & 1.99 & -7.3 & -7.7 \\
\hline 19 & 3.13 & 3.17 & -15.2 & -15.7 \\
\hline 20 & 2.54 & 2.57 & -3.4 & -3.9 \\
\hline 21 & 2.56 & 2.59 & -3.2 & -3.5 \\
\hline 22 & 3.15 & 3.18 & -17.2 & -18.2 \\
\hline 23 & 1.82 & 1.83 & -10.1 & -10.1 \\
\hline 24 & 3.12 & 3.16 & -16.8 & -16.5 \\
\hline 25 & 1.86 & 1.87 & -9.1 & -10.8 \\
\hline 26 & 2.22 & 2.20 & 0.6 & 0.3 \\
\hline 27 & 3.14 & 3.16 & -16.0 & -16.5 \\
\hline 28 & 3.36 & 3.30 & -9.9 & -11.2 \\
\hline 29 & 1.71 & 1.74 & -21.0 & -19.2 \\
\hline 30 & 1.81 & 1.80 & -15.3 & -15.1 \\
\hline 31 & 1.89 & 1.92 & -12.6 & -12.1 \\
\hline 32 & 1.92 & 1.95 & -17.0 & -16.1 \\
\hline 33 & 2.07 & 2.07 & -14.1 & -13.8 \\
\hline 34 & 1.94 & 1.91 & -17.8 & -16.8 \\
\hline
\end{tabular}


Table S3. Computational and experimental gas-phase energies along the decarboxylation reaction path.

\begin{tabular}{cccc}
\hline Index & $\Delta \mathrm{E}(\mathrm{expt})$ & $\Delta \mathrm{E}(\mathrm{M} 06-2 \mathrm{X})$ & $\Delta \mathrm{E}(\mathrm{AM} 1)$ \\
\hline 1 & $61.6 \pm 3.5^{a}$ & 66.4 & 93.3 \\
2 & $60.6 \pm 3.5^{a}$ & 55.9 & 64.5 \\
3 & - & 33.0 & 36.1 \\
4 & $40.1 \pm 4.6^{a}$ & 38.7 & 28.6 \\
5 & $34.1 \pm 4.6^{a}$ & 29.3 & 44.3 \\
6 & - & 91.5 & 113.7 \\
7 & $27.9 \pm 1.2^{b}$ & 22.0 & 33.3 \\
8 & $15.7 \pm 1.1^{b}$ & 18.9 & 19.7 \\
9 & - & 22.7 & 38.5 \\
10 & $24.7^{\mathrm{c}}$ & 21.2 & 19.7 \\
11 & - & 15.6 & 23.1 \\
\hline
\end{tabular}

${ }^{a}$ Reference 1 .

${ }^{b}$ Reference 2 .

'Reference 3, calculated at MP2/6-31+g(d) level.

Table S4 Optimized values of the Lennard-Jones (LJ) rmin and $\varepsilon$ parameter for the description of the vDW interaction between representative atom pairs in Figure 1 . All $r_{\min }$ are given in $\AA$ and $\varepsilon$ are given in $\mathrm{kcal} / \mathrm{mol}$.

\begin{tabular}{ccc}
\hline Atom & $\sigma(\AA)$ & $\varepsilon(\mathrm{kcal} / \mathrm{mol})$ \\
\hline $\mathrm{O}$ & 1.61 & \\
$\mathrm{~N}$ & 1.74 & \\
$\mathrm{H}$ & 0.33 & \\
$\mathrm{C} 1^{-} / \mathrm{C}^{-}$ & 1.53 & \\
$\mathrm{C}^{-} / \mathrm{C}^{-} / \mathrm{C5}^{-}$ & 1.70 & \\
\hline
\end{tabular}

1. Belsky, A.J., P.G. Maiella, and T.B. Brill, Spectroscopy of hydrothermal reactions 13. kinetics and mechanisms of decarboxylation of acetic acid derivatives at 100-260 ${ }^{\circ} \mathrm{C}$ under 275 bar. Journal of Physical Chemistry A, 1999. 103: p. 4253-4260.

2. Maiella, P.G. and T.B. Brill, Spectroscopy of Hydrothermal Reactions. 5. Decarboxylation Kinetics of Malonic Acid and Monosodium Malonate. J. Phys. Chem., 1996. 100: p. 14352-14355.

3. Gao, D. and Y.-K. Pan, A QM/MM monte carlo simulation study of solvent effects on the decarboxylation reaction of $N$ carboxy-2-imidazolidinone anion in aqueous solution. Journal of Organic Chemistry, 1999. 64: p. 1151-1159. 
Table S5. Estimation of free energies of reaction for the decarboxylation of N-methylorotate and nitroacetate in water based on experimental acidity constants $\mathrm{pKa}$ and computed free energy difference of the corresponding decarboxylation of the neutral acids. Considering the following thermodynamic cycle,

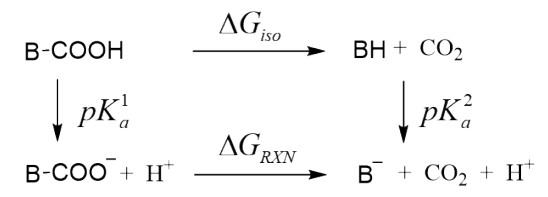

we relate the Gibbs energy of reaction to the difference in $\mathrm{pKa}$ for the carboxylic acid and the conjugate acid of the decarboxylation product plus an isomerization free energy term corresponding to a decarboxylation of the neutral acid.

$$
\Delta G_{R X N}=2.303 R T\left(p K_{a}^{2}-p K_{a}^{1}\right)+\Delta G_{i s o}
$$

The experimental values for the $\mathrm{pKa}$ are used and the free energy of transfer are determined by using M06-2X/aug-cc-pVTZ for the gas phase process along with solvation free energies from the SMD model with the Gaussian16 package. Solvation energies are determined by using B3LYP/aug-cc-pVDZ using M06-2X optimized structures. The electronic energies for $\mathrm{CO}_{2}$. The isomerization results are summarized below, and all structures are listed in this supporting information. Total energies are given in a.u., and relative energies in $\mathrm{kcal} / \mathrm{mol}$.

\begin{tabular}{|l|c|c|c|c|}
\hline & Orotic acid & Uracil $+\mathrm{CO}_{2}$ & Nitroacetic acid & Nitromethane $+\mathrm{CO}_{2}$ \\
\hline$G_{\text {elec }}^{298}$ & -642.61576 & -642.64790 & -433.55446 & -433.58728 \\
\hline$G_{\text {elec }}^{\text {SMD }}$ & -642.81035 & -642.82071 & -433.66325 & -433.66906 \\
\hline$E_{\text {elec }}^{\text {gas }}$ & -642.78775 & -642.80655 & -433.64823 & -433.66728 \\
\hline$\Delta G_{\text {elec }}^{\text {SMD }}$ & -14.2 & -8.9 & -9.4 & -1.1 \\
\hline$\Delta G_{\text {CDS }}^{\text {SMD }}$ & 7.0 & 11.1 & 6.7 & 10.6 \\
\hline$\Delta G_{\text {solv }}^{\text {SMD }}$ & -7.2 & 2.2 & -2.7 & 9.5 \\
\hline$\Delta \Delta G_{\text {solv }}^{\text {SMD }}$ & 0.0 & 9.3 & 0.0 & 12.1 \\
\hline$\Delta G_{\text {elec }}^{298}$ & 0.0 & -20.2 & 0.0 & -20.6 \\
\hline $\mathrm{pKa}$ & 34 & 2.1 & 1.7 & 10.2 \\
\hline$\Delta G_{\text {iso }}$ & 0.0 & 32.6 & 0.0 & 3.1 \\
\hline
\end{tabular}


Table S6. The decomposition of the QM/MM interaction energy of reactant, transition and product state. Their errors are approximately $\pm 1.2 \mathrm{kcal} / \mathrm{mol}$ for $\Delta E_{X s}^{\text {elec }}$ and $\pm 0.3 \mathrm{kcal} / \mathrm{mol}$ for $\Delta E_{X s}^{\text {pol }}$ (1. Acetate anion; 2. Benzoate anion; 3. N-Methylorotate anion; 4. Trifluoroacetate anion; 5. Cyanoacetate anion; 6. Malonate dianion; 7. Malonate monoanion; 8. Trichloroacetate anion; 9. Acetoacetate anion; 10. oxoimidazolidine-1-carboxylate ion; and 11. Nitroacetate anion). The energy components that contribute to the total solute-solvent interactions for each decarboxylation reaction have been obtained at the reactant conformation, the transition state and the product state at an RC of $5 \AA$.

\begin{tabular}{|c|c|c|c|c|c|c|}
\hline NO. & $\Delta E_{X s}^{\text {elec }}$ & $\Delta E_{X s}^{o}$ & $\Delta E_{X s}^{p o l}$ & $\Delta E_{X s}^{\text {gain }}$ & $\Delta E_{X s}^{d i s t}$ & $\Delta E_{v d W}$ \\
\hline \multicolumn{7}{|c|}{$\mathrm{RS}$} \\
\hline 1 & -187.5 & -182.5 & -5.0 & -9.9 & 4.9 & -3.3 \\
\hline 2 & -190.2 & -177.8 & -12.3 & -24.5 & 12.1 & -11.0 \\
\hline 3 & -161.9 & -155.5 & -6.4 & -12.7 & 6.2 & -19.5 \\
\hline 4 & -154.1 & -151.6 & -2.5 & -5.0 & 2.5 & -3.2 \\
\hline 5 & -172.9 & -167.8 & -5.0 & -9.9 & 4.9 & -12.7 \\
\hline 6 & -540.1 & -535.6 & -4.5 & -8.8 & 4.4 & -2.3 \\
\hline 7 & -193.1 & -185.0 & -8.1 & -16.0 & 7.9 & -11.6 \\
\hline 8 & -160.9 & -155.9 & -5.0 & -9.8 & 4.8 & -5.1 \\
\hline 9 & -207.2 & -197.4 & -9.9 & -19.5 & 9.7 & -4.0 \\
\hline 10 & -214.4 & -199.8 & -14.6 & -29.1 & 14.5 & -4.9 \\
\hline 11 & -170.2 & -165.3 & -4.9 & -9.6 & 4.7 & -5.1 \\
\hline \multicolumn{7}{|c|}{ TS } \\
\hline 1 & -141.8 & -139.6 & -2.1 & -4.3 & 2.1 & -2.6 \\
\hline 2 & -152.4 & -143.3 & -9.1 & -18.3 & 9.2 & -9.1 \\
\hline 3 & -132.4 & -128.1 & -4.3 & -8.5 & 4.2 & -16.7 \\
\hline 4 & -135.4 & -134.7 & -0.8 & -1.5 & 0.8 & -0.2 \\
\hline 5 & -132.5 & -130.3 & -2.2 & -4.5 & 2.2 & -15.7 \\
\hline 6 & -524.7 & -519.7 & -5.0 & -10.0 & 5.0 & -2.9 \\
\hline 7 & -152.3 & -148.0 & -4.3 & -8.6 & 4.3 & -13.3 \\
\hline 8 & -139.5 & -136.3 & -3.1 & -6.3 & 3.1 & -11.4 \\
\hline 9 & -156.7 & -150.6 & -6.1 & -12.2 & 6.1 & -8.0 \\
\hline 10 & -166.5 & -157.6 & -9.0 & -17.9 & 8.9 & -10.7 \\
\hline 11 & -145.5 & -142.7 & -2.9 & -5.8 & 2.9 & -8.7 \\
\hline \multicolumn{7}{|c|}{ PS } \\
\hline 1 & -181.2 & -170.5 & -10.8 & -16.0 & 5.3 & -0.8 \\
\hline 2 & -140.7 & -135.5 & -5.2 & -10.3 & 5.1 & -8.7 \\
\hline 3 & -145.6 & -140.7 & -4.9 & -9.7 & 4.8 & -19.4 \\
\hline 4 & -148.8 & -147.0 & -1.8 & -3.2 & 1.3 & -0.4 \\
\hline 5 & -157.9 & -153.9 & -4.0 & -7.5 & 3.4 & -14.6 \\
\hline 6 & -594.9 & -568.1 & -26.8 & -37.5 & 10.7 & -0.9 \\
\hline 7 & -167.9 & -163.9 & -4.0 & -7.7 & 3.8 & -14.4 \\
\hline 8 & -205.6 & -198.9 & -6.7 & -13.3 & 6.6 & -10.2 \\
\hline 9 & -170.7 & -164.2 & -6.5 & -12.6 & 6.1 & -7.4 \\
\hline 10 & -167.0 & -158.2 & -8.9 & -17.5 & 8.6 & -9.1 \\
\hline 11 & -169.8 & -164.9 & -4.9 & -9.8 & 4.9 & -6.5 \\
\hline
\end{tabular}


Figure S1. Some geometry parameters of the optimized equilibrium structures obtained at high-level M06-2X/aug-cc-pVTZ and low-level semiempirical AM1 method (in brackets).
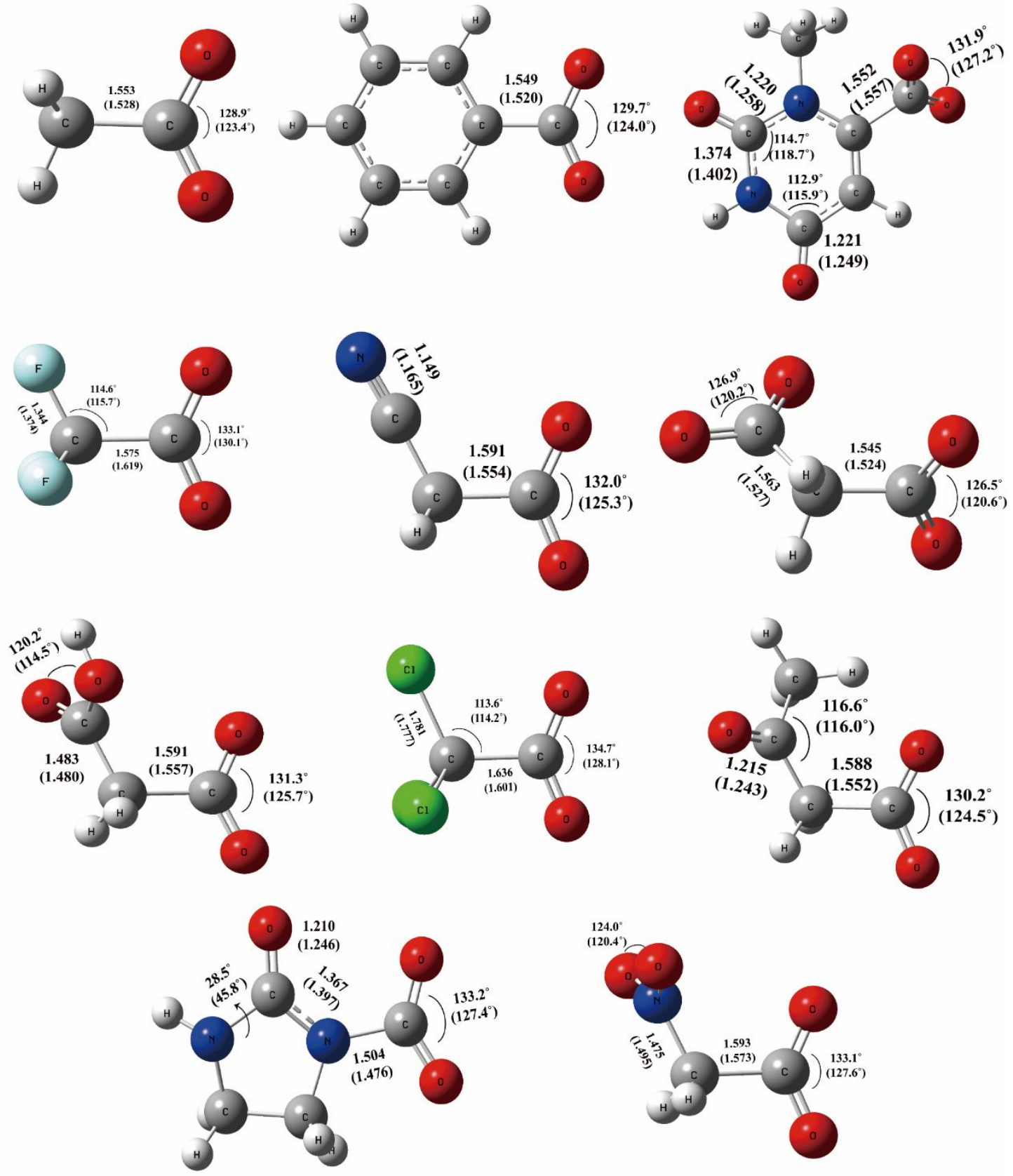
Figure S2. Bimolecular complexes along with computed binding energies and optimized hydrogen bond distance for carboxylate anions (A) and carbanions (B) with a water molecule at different interaction sites. Results from M06-2X density functional theory calculations are given first, followed by QM/MM results in parentheses. Atom types in red are optimized. Distances are in angstroms and energies are given in kilocalories per mole.

(A)

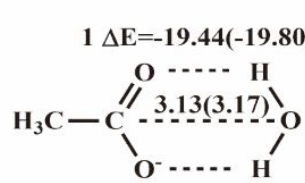
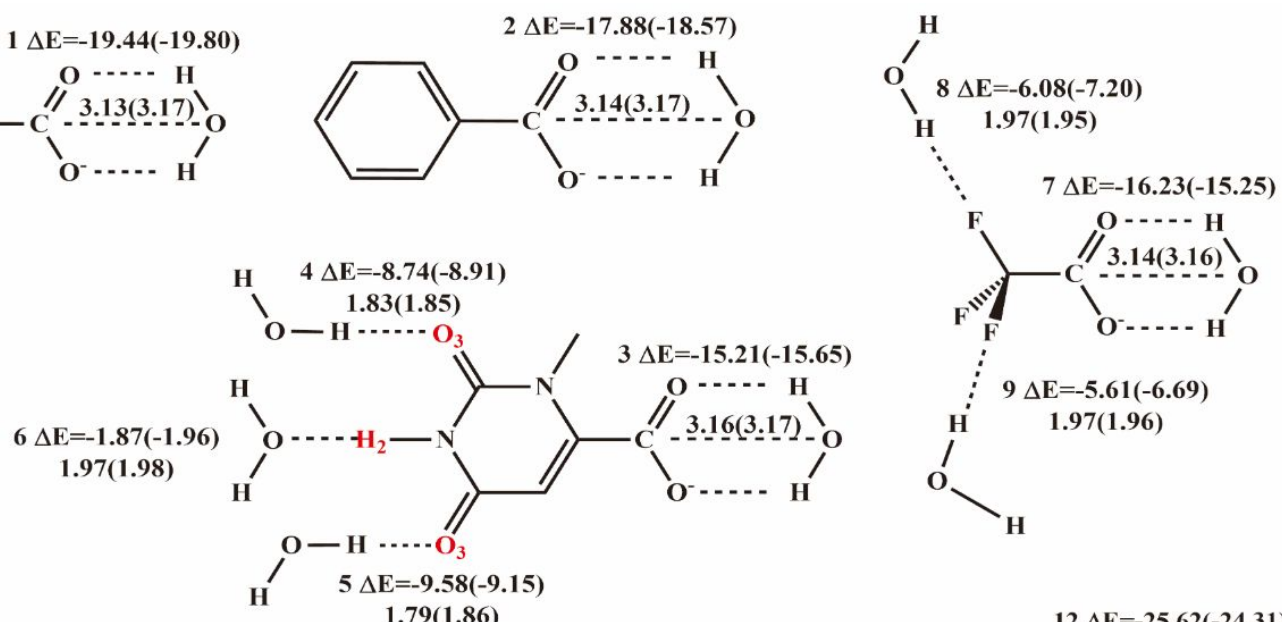<smiles>OI</smiles>
1.79(1.86)
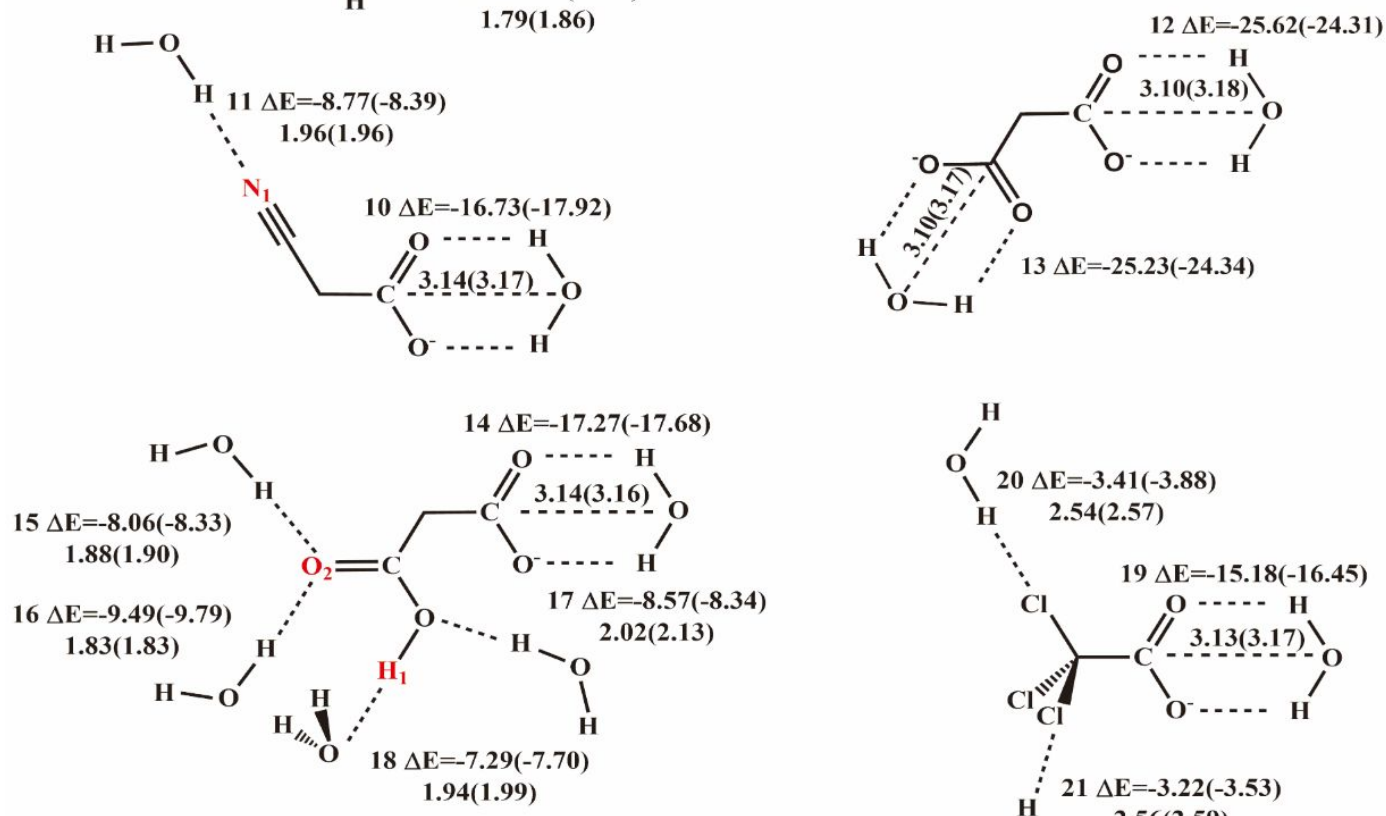

$11 \Delta E=-8.77(-8.39)$

$1.96(1.96)$<smiles>[AlH2]=[V]</smiles>

$10 \Delta \mathrm{E}=-16.73(-17.92)$<smiles></smiles>
- H

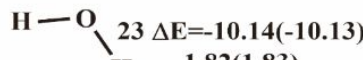

H $1.82(1.83)$

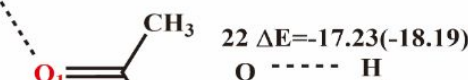

H $2.54(2.57)$<smiles>CCCCC</smiles>
$\because \quad 19 \Delta \mathrm{E}=-15.18(-16.45)$

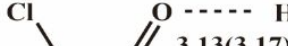<smiles>C=C([C@@H](C)Cl)C(Cl)(Cl)CC</smiles>

$\therefore$

$21 \Delta \mathrm{E}=-3.22(-3.53)$

H $2.56(2.59)$

$\stackrel{\text { O }}{{ }^{\prime}}$ // $\mathrm{O}^{-} \ldots .$.

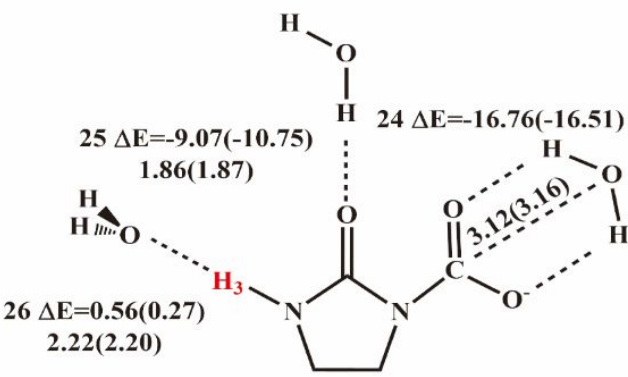


(B).
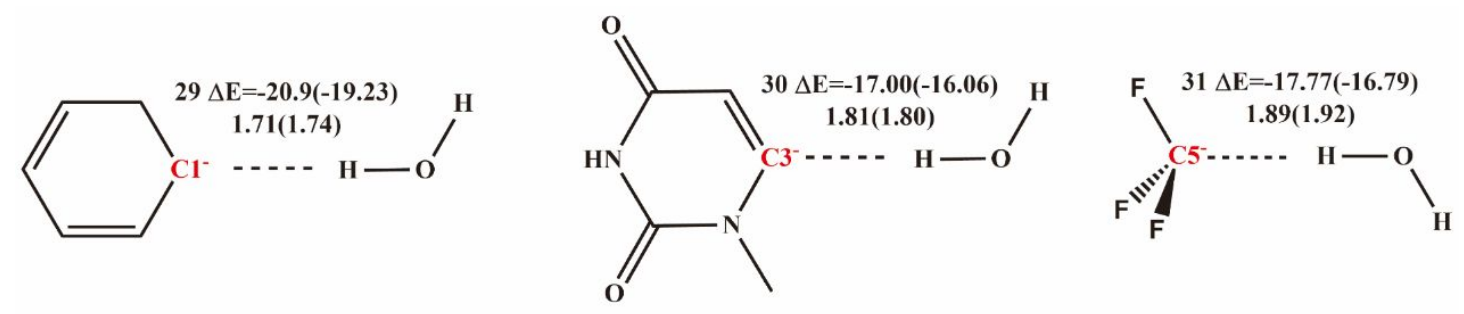<smiles>O</smiles>
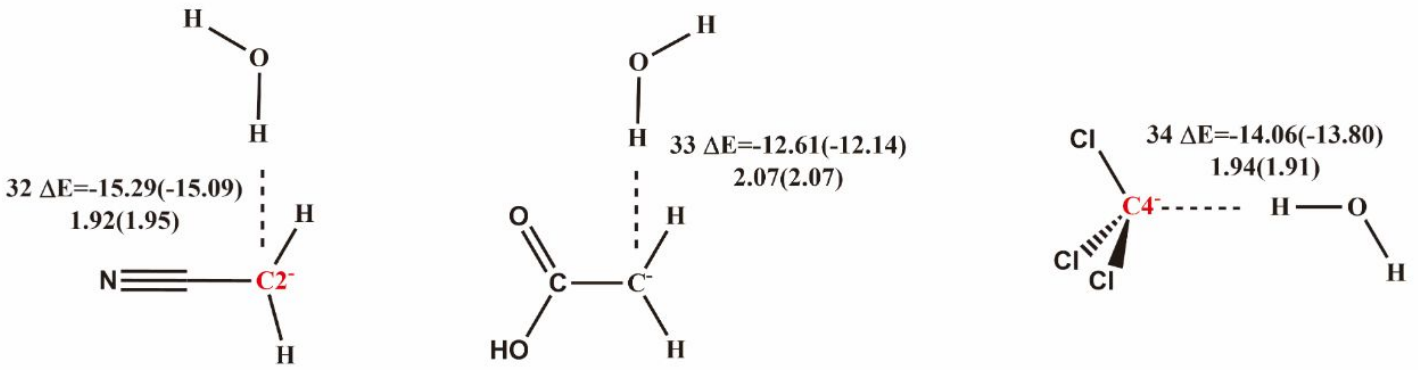
Figure S3. Energy terms in Eq. 10 and variations in solvation free energy along the reaction path and the final corrected PMFs of along the decarboxylation reaction coordinates. (all of the energy points along these reactions are given in the Excel document).
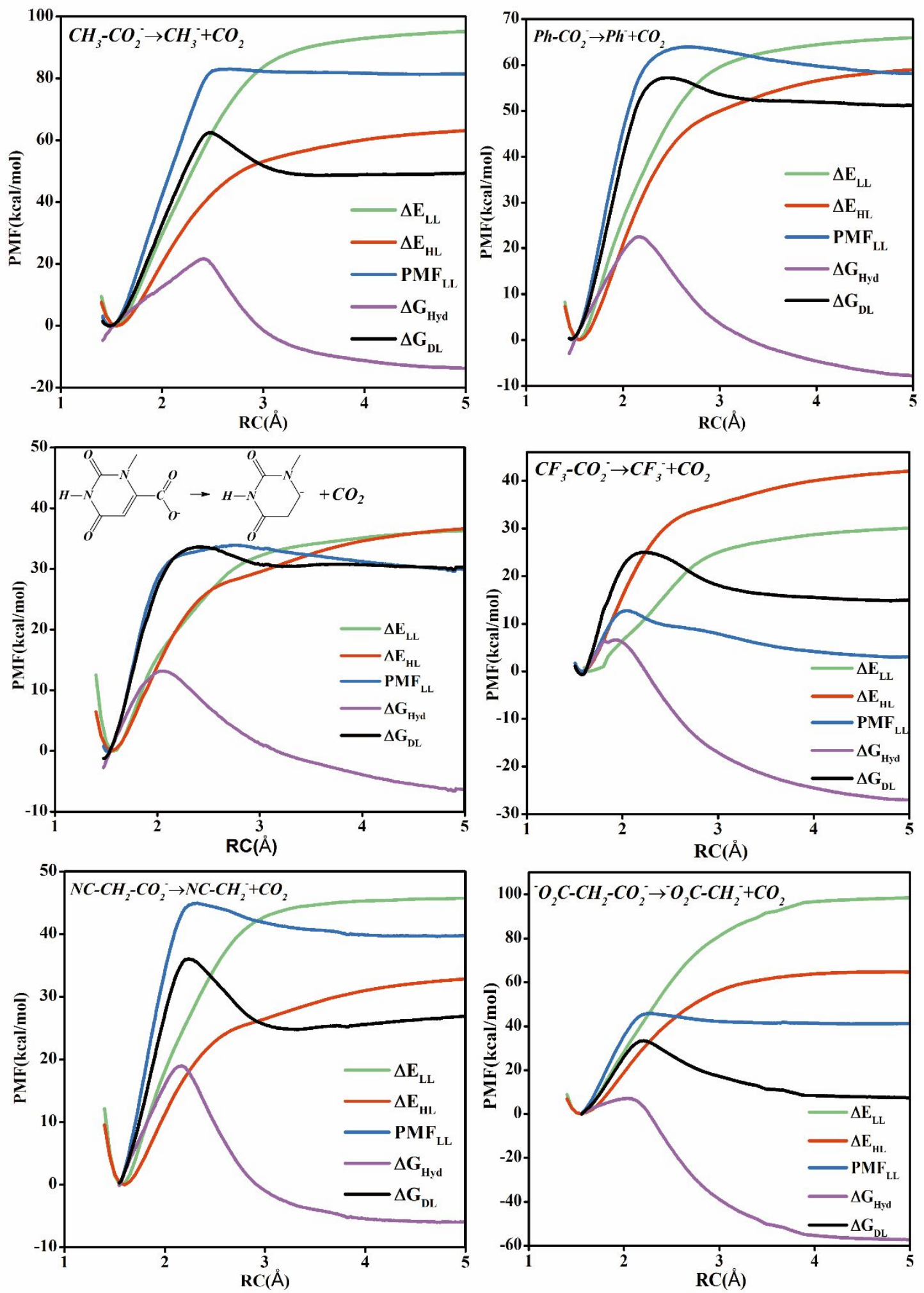
Figure S3. Continued.
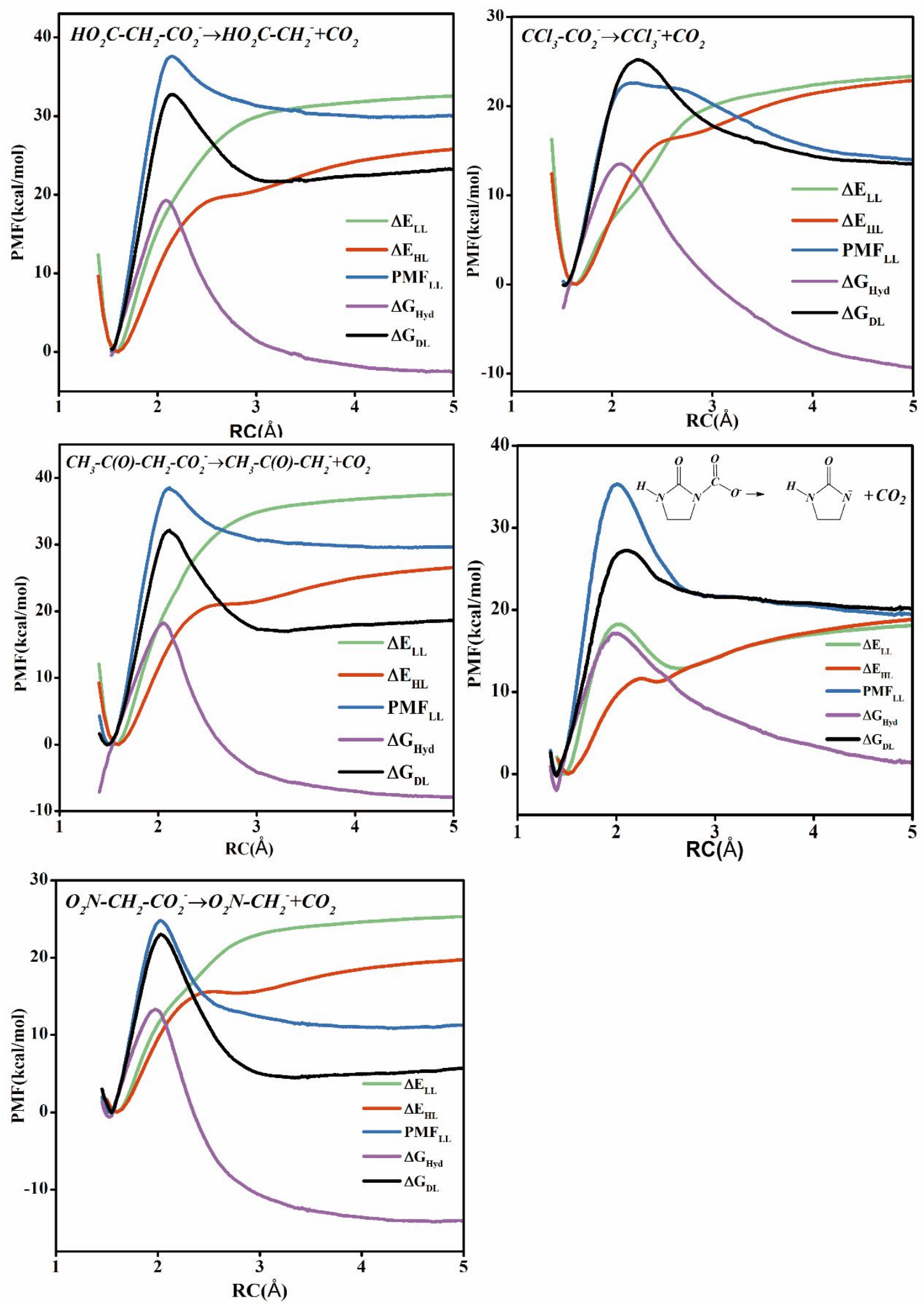
Figure S4. Correlation between calculated $\Delta E_{X s}^{\text {elec }}$ and $\Delta E_{X s}^{\text {pol }}$.

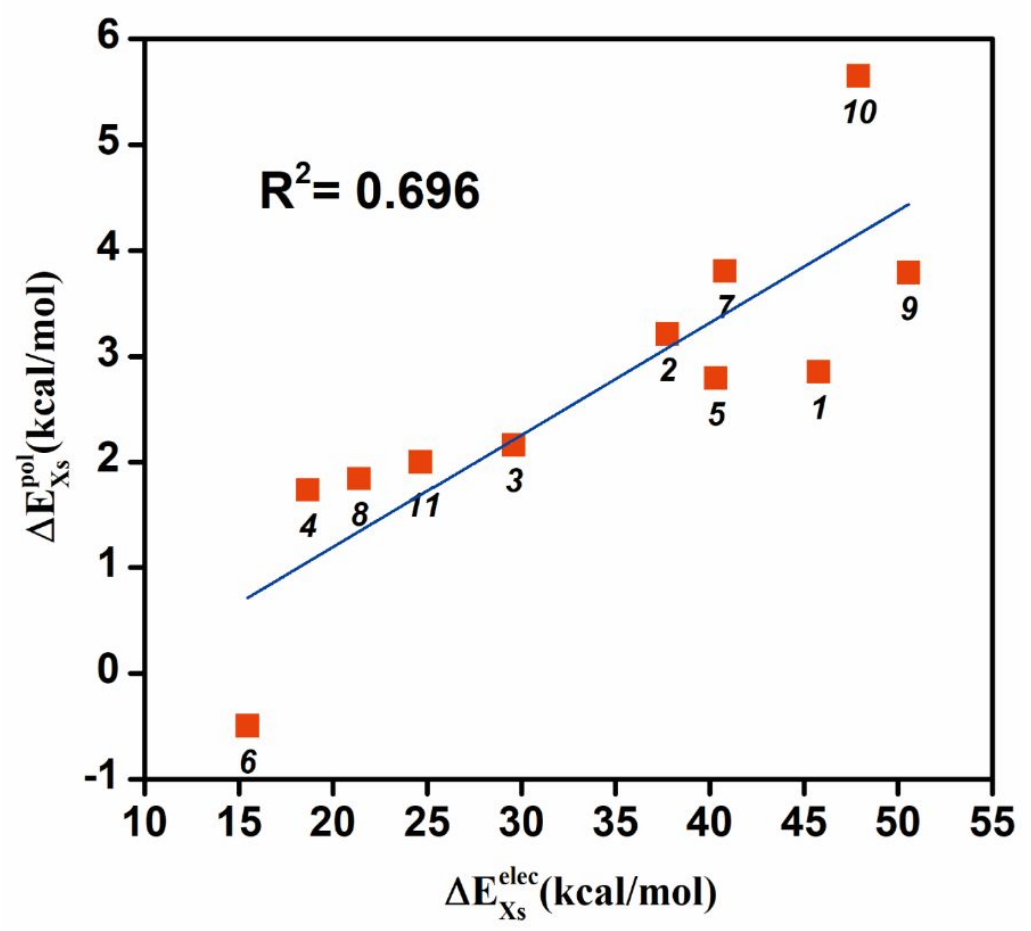

Table S6 shows that for the ionic systems considered here, the computed energies needed for the solute to rearrange its wave function in response to the electrostatic polarization by the solvent molecules, $\Delta E_{X}^{\text {dist }}$, are about one-half of the energy gain of solute-solvent interactions $\Delta E_{X s}^{\text {gain }}$, in accord with classic linear response theory. We found that the magnitudes of electronic polarization of the solute molecules are depesndent on the electron-withdrawing strength of the functional groups that host the carboxylate group, ranging from 8 to $16 \%$ of the total solute-solvent interaction energy. Overall, electronegative groups such as $-\mathrm{NO}_{2},-\mathrm{CN}$, and $-\mathrm{F}$ tend to have weaker polarization effects since the charge density is more strongly held than electron donating groups in the substrate. Furthermore, polarization effects also correlate with the strength of solute-solvent interaction energies due to intrinsic (gas phase) charge distribution (Figure S5). 
Figure S5-1. Radial distribution functions (RDFs) for $\mathrm{Ph}_{-} \mathrm{CO}_{2}-\mathrm{C} 2-\mathrm{HW}$ and $\mathrm{C} 2-\mathrm{OW}$ pairs at different states.
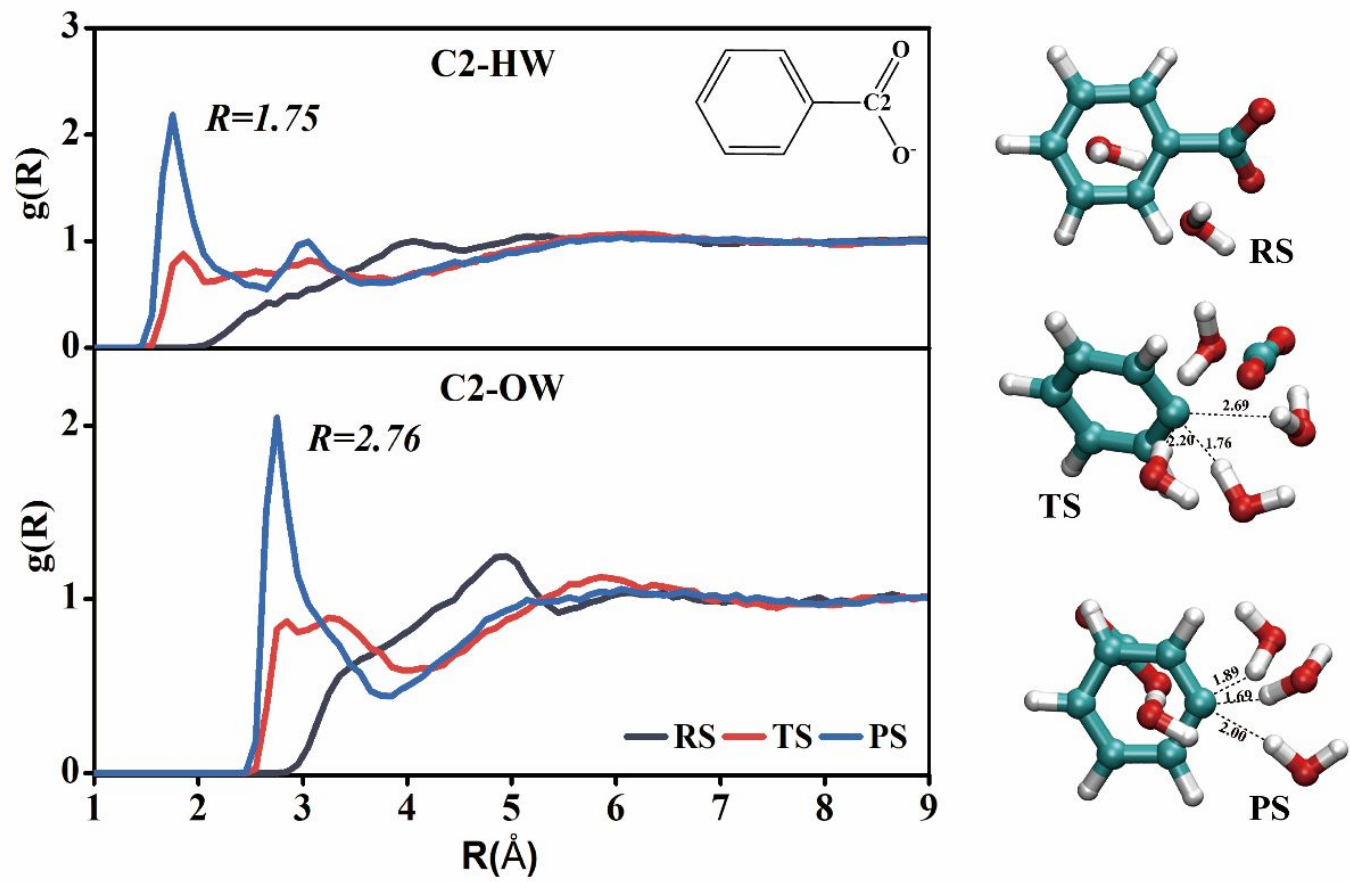

Figure S5-2. $\mathrm{RDFs}$ for $\mathrm{CN}_{-}-\mathrm{CH}_{2}-\mathrm{CO}_{2}-\mathrm{N}-\mathrm{HW}$ and $\mathrm{C} 2-\mathrm{HW}$ pairs at different states.
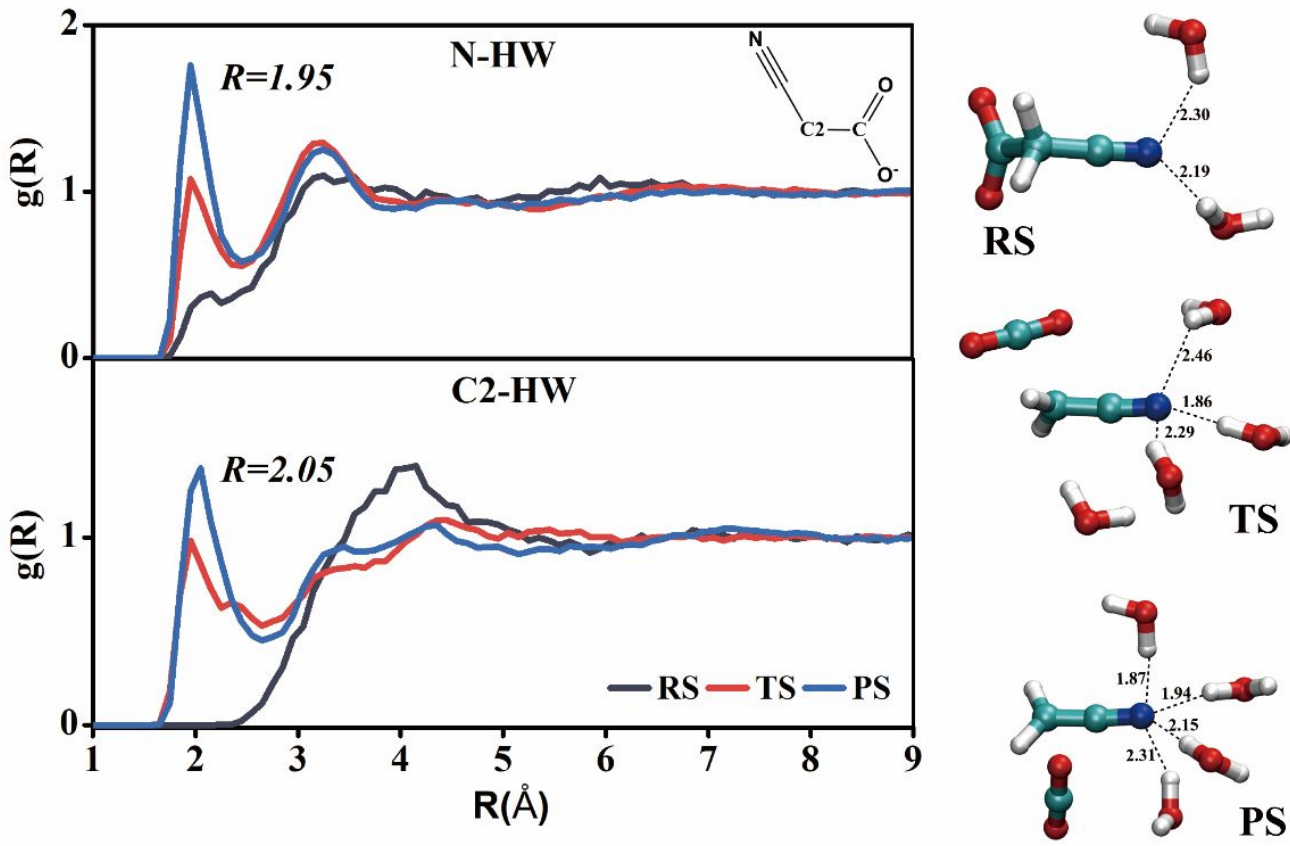
Figure S5-3. RDFs for $\mathrm{COOH}-\mathrm{CH}_{2}-\mathrm{CO}_{2}^{-}: \mathrm{O} 3-\mathrm{HW}$ and $\mathrm{H}-\mathrm{OW}$ pairs at different states.
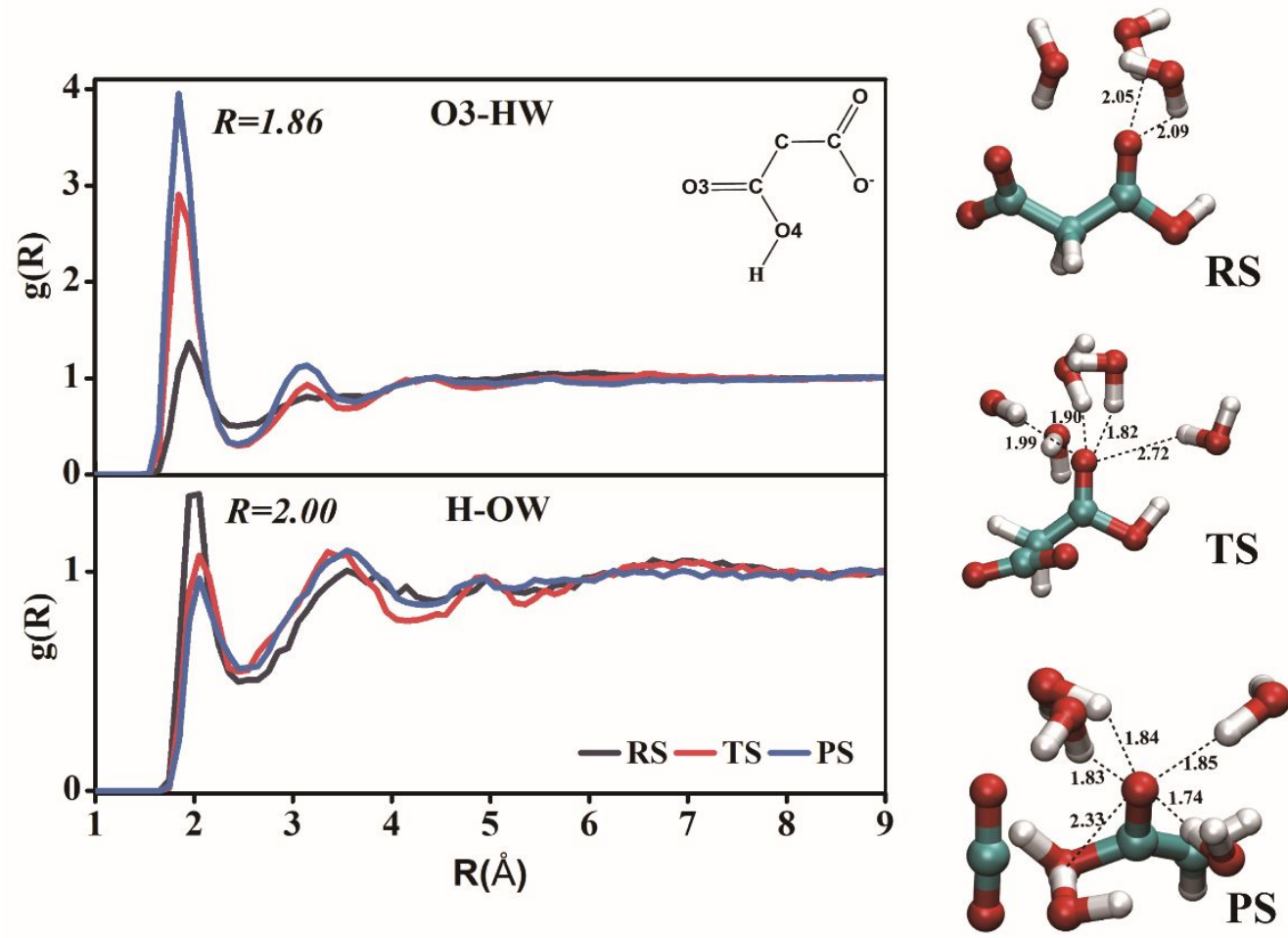

Figure S5-4. RDFs for $\mathrm{NO}_{2}-\mathrm{CH}_{2}-\mathrm{CO}_{2}^{-:} \mathrm{O} 1-\mathrm{HW}$ and $\mathrm{O} 3-\mathrm{HW}$ at different states.
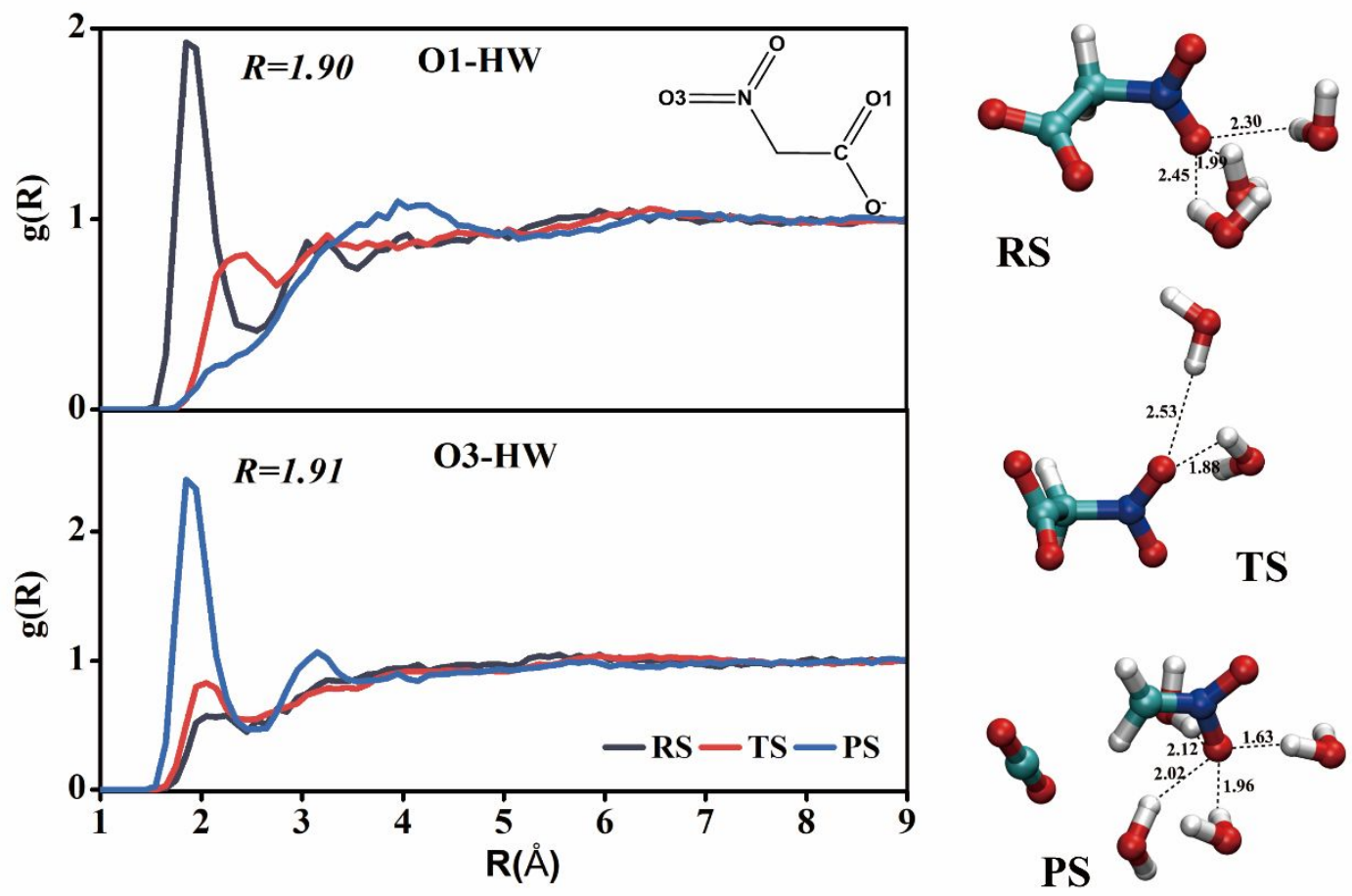
Figure S5-5. RDFs for $\mathrm{Cl}_{3}-\mathrm{CO}_{2}^{-}{ }^{-} \mathrm{C} 2-\mathrm{HW}$ and $\mathrm{Cl}-\mathrm{HW}$ at different states.
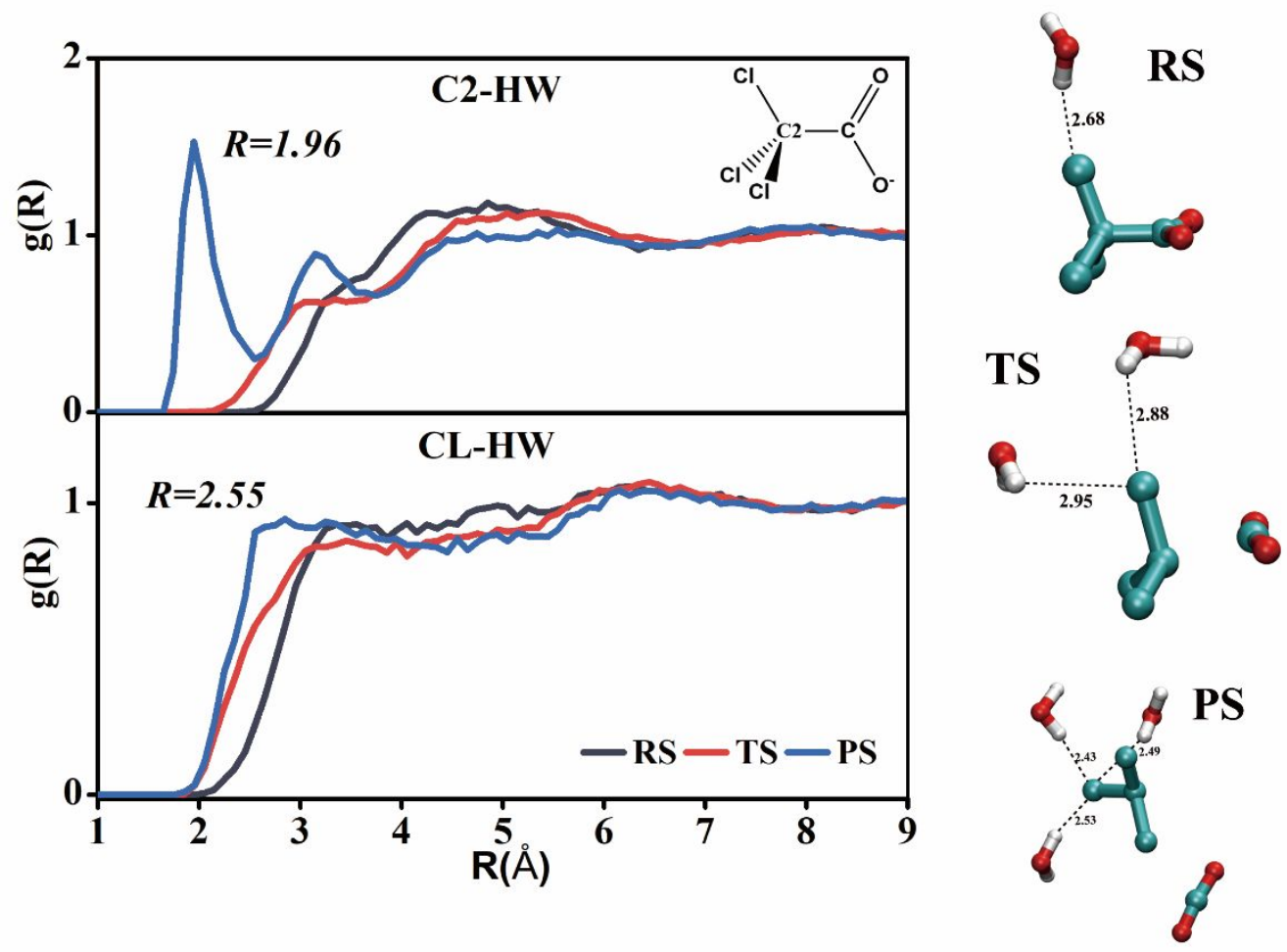

Figure S5-6. RDFs for $\mathrm{F}_{3}-\mathrm{CO}_{2}^{-}: \mathrm{C} 2-\mathrm{HW}$ and $\mathrm{F}-\mathrm{HW}$ at different states.
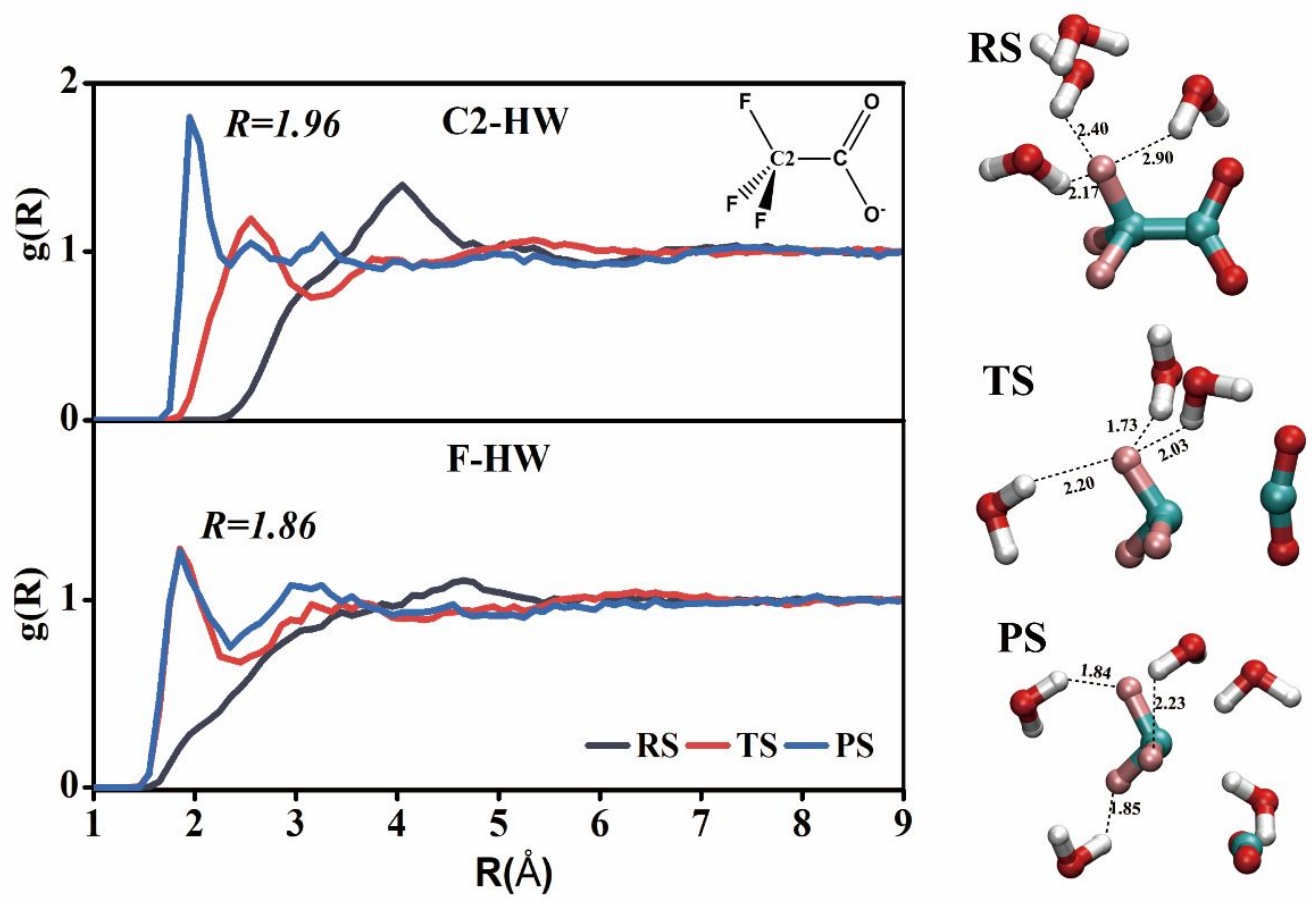
Appendix-Z-matrix of 34 hydrogen bond interaction complexes

Complex 1:

$-11$

C

C,1,B1

$\mathrm{O}, 1, \mathrm{~B} 2,2, \mathrm{~A} 1$

$\mathrm{O}, 1, \mathrm{B3}, 3, \mathrm{~A} 2,2, \mathrm{D} 1,0$

$\mathrm{H}, 2, \mathrm{~B} 4,1, \mathrm{~A} 3,3, \mathrm{D} 2,0$

$\mathrm{H}, 2, \mathrm{B5}, 1, \mathrm{~A} 4,3, \mathrm{D3}, 0$

H,2,B6,1,A5,3,D4,0

$\mathrm{O}, 1, \mathrm{~B} 7,4, \mathrm{~A} 6,2, \mathrm{D} 5,0$

$\mathrm{H}, \mathbf{8 , B 8 , 1 , A 7 , 4 , D 6 , 0}$

H,8,B9,9,A8,1,D7,0

Variables:

B7=3.13267989

Constants:

$B 1=1.55271784$

$B 2=1.24928188$

$B 3=1.24928188$

B4=1.08994052

$B 5=1.08994052$

$B 6=1.09296865$

$B 8=0.9672$

B9 $=0.9672$

$A 1=115.56590543$

A2 $=\mathbf{1 2 8 . 8 5 1 6 0 2 6 7}$

A3 $=110.88345393$

A4 $=110.88345393$

A5 $=\mathbf{1 0 8 . 1 8 6 1 5 4 0 7}$

A6 $=64.4472$

$\mathrm{A} 7=\mathbf{5 2 . 2 6}$

A8 $=104.52$

D1 $=178.43776598$

D2 $=-\mathbf{2 8 . 9 5 9 5 8 6 8 7}$

D3 $=-\mathbf{1 5 2 . 3 8 9 0 4 6 2 3}$

D4 $=89.32568345$

D5 $=\mathbf{- 1 8 0}$.

D6 $=0$.

D7 $=$ 0.

Complex 2:

$-11$

C

C,1,B1

O,1,B2,2,A1

$\mathrm{O}, 1, \mathrm{B3}, 3, \mathrm{A2}, 2, \mathrm{D} 1,0$

$\mathrm{C}, 2, \mathrm{~B} 4,1, \mathrm{A3}, 4, \mathrm{D} 2,0$

$\mathrm{C}, 2, \mathrm{B5}, 1, \mathrm{A4}, 4, \mathrm{D3}, 0$

$\mathrm{C}, 5, \mathrm{~B} 6,2, \mathrm{A5}, 1, \mathrm{D} 4,0$

H,5,B 7,2,A6, 1,D5,0

$\mathrm{C}, 6, \mathrm{B8}, 2, \mathrm{~A} 7,1, \mathrm{D6}, 0$

H,6,B9,2,A8,1,D7,0

C,9,B10,6,A9,2,D8,0

H,7,B11,5,A10,2,D9,0

H,9,B12,6,A11,2,D10,0

H,11,B13,9,A12,6,D11,0 
O,1,B14,3,A13,2,D12,0

H,15,B15,1,A14,3,D13,0

H,15,B16,1,A15,4,D14,0

Variables:

B14=3.14276713

Constants:

B1 $=1.549148$

$B 2=1.24568972$

$B 3=1.24568972$

$B 4=1.3908856$

B5 $=1.3908856$

B6 $=1.38929675$

$B 7=1.08216085$

$B 8=1.38929675$

B9 $=1.08216085$

$\mathrm{B} 10=1.3901672$

B11 $=\mathbf{1 . 0 8 3 7 0 5 6 7}$

$B 12=1.08370567$

B13 $=1.083252$

$B 15=0.96720023$

$B 16=0.96720023$

A1 $=115.14547403$

A2 $=129.70905189$

$\mathrm{A} 3=\mathbf{1 2 0 . 6 6 1 2 6 0 2}$

A4 $=120.6612602$

A5 $=120.83979213$

A6 $=117.25297249$

A7 $=\mathbf{1 2 0 . 8 3 9 7 9 2 1 4}$

A8 $=117.25297249$

A9 $=120.08053082$

$A 10=120.1200739$

A11 $=120.1200739$

$A 12=120.25906275$

$A 13=64.854525$

A14 $=52.26$

$\mathbf{A 1 5}=\mathbf{5 2 . 2 6}$

D1 $=-179.9969141$

D2 $=-\mathbf{0 . 0 0 0 7 7 3 9}$

D3 $=179.9983009$

D4 $=179.99935951$

D5 $=-\mathbf{0 . 0 0 0 8 3 4 1 1}$

D6 $=-179.99935933$

D7 $=\mathbf{0 . 0 0 0 8 4 8 0 3}$

D8 $=0$.

D9=180.

D10 $=-180$.

D11 $=180$.

D12 $=180$.

D13 $=$. .

D14 $=0$.

Complex 3:

$-11$

C

C,1,B1

$\mathrm{O}, 1, \mathrm{~B} 2,2, \mathrm{~A} 1$

$\mathrm{O}, 1, \mathrm{B3}, 3, \mathrm{~A} 2,2, \mathrm{D} 1,0$

$\mathrm{C}, 2, \mathrm{~B} 4,1, \mathrm{~A} 3,4, \mathrm{D} 2,0$

$\mathrm{N}, 5, \mathrm{B5}, 2, \mathrm{~A} 4,1, \mathrm{D3}, 0$ 
C,2,B6,1,A5,4,D4,0

$\mathrm{C}, 7, \mathrm{~B} 7,2, \mathrm{A6,1,D5,0}$

N,5,B8,2,A7,1,D6,0

H,7,B9,2,A8,1,D7,0

H,9,B10,5,A9,2,D8,0

C,6,B11,5,A10,2,D9,0

H,12,B12,6,A11,5,D10,0

H,12,B13,6,A12,5,D11,0

H,12,B14,6,A13,5,D12,0

O,5,B15,2,A14,1,D13,0

O,8,B16,7,A15,2,D14,0

O,1,B17,3,A16,2,D15,0

H,18,B18,1,A17,3,D16,0

H,18,B19,19,A18,4,D17,0

Variables:

B17 $=3.15705234$

Constants:

$B 1=1.55178421$

$B 2=1.24182114$

$B 3=1.23535246$

B4=2.40906672

$B 5=1.37732874$

B6 $=\mathbf{1 . 3 5 1 7 6 7 7 7}$

$\mathrm{B} 7=\mathbf{1 . 4 3 4 7 3 2 5 1}$

$B 8=1.37428629$

B9=1.07723985

$\mathrm{B} 10=\mathbf{1 . 0 0 8 3 3 2 2 5}$

$B 11=1.46281973$

$B 12=1.08465789$

$B 13=1.08670697$

B14 $=1.08529274$

$B 15=1.21997852$

B16 $=1.22071407$

$\mathrm{B} 18=\mathbf{0 . 9 5 7 2}$

$\mathrm{B} 19=0.9572$

A1 $=113.03957519$

A2 $=131.94153345$

A3 $=146.55041073$

A4 $=29.14472589$

A5 $=120.55297521$

A6 $=120.94699292$

$\mathrm{A} 7=\mathbf{8 5 . 4 8 9 7 0 9 1}$

$A 8=119.69565688$

A9 $=115.27937451$

$\mathrm{A10}=\mathbf{1 1 7 . 0 4 0 8 6 2 4 5}$

$\mathrm{A} 11=\mathbf{1 0 7 . 6 6 5 0 4 3 7 7}$

A12 $=\mathbf{1 1 0 . 3 9 7 3 0 0 6}$

$A 13=108.91093154$

A14 $=153.06943363$

$A 15=127.96703226$

$\mathrm{A} 16=\mathbf{6 5 . 9 7 0 7 6 5}$

$\mathrm{A17}=\mathbf{5 2 . 2 6}$

A18 $=104.52$

D1=-178.53818219

D2 $=-133.87033211$

D3 $=9.53425828$

D4 $=\mathbf{5 4 . 7 6 6 2 0 0 6 2}$

D5 $=174.31300511$

D6 $=-171.39120507$

D7 $=-5.75806162$ 


$$
\begin{aligned}
& D 8=179.52131799 \\
& D 9=179.29204232 \\
& D 10=-23.05714726 \\
& D 11=97.90186 \\
& D 12=-143.40314511 \\
& D 13=8.30144484 \\
& D 14=-180 . \\
& D 15=-180 . \\
& \text { D16 }=0 . \\
& \text { D17 }=0 .
\end{aligned}
$$

Complex 4:

-11
$C$

$$
\text { C,1,B1 }
$$

$\mathrm{O}, 1, \mathrm{~B} 2,2, \mathrm{~A} 1$

$\mathrm{O}, 1, \mathrm{B3}, 3, \mathrm{~A} 2,2, \mathrm{D} 1,0$

$\mathrm{C}, 2, \mathrm{~B} 4,1, \mathrm{A3}, 4, \mathrm{D} 2,0$

$\mathrm{N}, 5, \mathrm{B5}, 2, \mathrm{~A} 4,1, \mathrm{D3}, 0$

$\mathrm{C}, 2, \mathrm{~B} 6,1, \mathrm{A5}, 4, \mathrm{D} 4,0$

$\mathrm{C}, 7, \mathrm{~B} 7,2, \mathrm{A6}, 1, \mathrm{D5}, 0$

N,5,B8,2,A7,1,D6,0

H,7,B9,2,A8,1,D7,0

H,9,B10,5,A9,2,D8,0

$\mathrm{C}, 6, \mathrm{~B} 11,5, \mathrm{A10}, 2, \mathrm{D9}, 0$

H,12,B12,6,A11,5,D10,0

H,12,B13,6,A12,5,D11,0

H,12,B14,6,A13,5,D12,0

$\mathrm{O}, 5, \mathrm{~B} 15,2, \mathrm{A14}, 1, \mathrm{D} 13,0$

O,8,B16,7,A15,2,D14,0

H,16,B17,5,A16,9,D15,0

$\mathrm{X}, 18, \mathrm{~B} 18,16, \mathrm{A1} 17,5, \mathrm{D} 16,0$

O,18,B19,19,A18,16,D17,0

H,20,B20,18,A19,19,D18,0 Variables:

B17 $=1.82651395$

A16=121.43014638 Constants:

B1 $=1.55178421$

$B 2=1.24182114$

$B 3=1.23535246$

B4 $=2.40906672$

B5 $=1.37732874$

B6 $=1.35176777$

B7 $=1.43473251$

$B 8=1.37428629$

B9 $=1.07723985$

$B 10=1.00833225$

$B 11=1.46281973$

B12 $=1.08465789$

B13 $=1.08670697$

B14 $=1.08529274$

$B 15=1.21997852$

$B 16=1.22071407$

B18 $=1$.

$\mathrm{B} 19=0.9572$

$\mathrm{B} 20=0.9572$

A1 $=113.03957519$

A2 $=131.94153345$ 


$$
\begin{aligned}
& A 3=146.55041073 \\
& \text { A4 }=29.14472589 \\
& \text { A5 }=\mathbf{1 2 0 . 5 5 2 9 7 5 2 1} \\
& A 6=120.94699292 \\
& \text { A7 }=\mathbf{8 5 . 4 8 9 7 0 9 1} \\
& A 8=119.69565688 \\
& \text { A9 }=115.27937451 \\
& A 10=117.04086245 \\
& \text { A11 }=107.66504377 \\
& \text { A12 }=110.3973006 \\
& \text { A13 }=108.91093154 \\
& \text { A14 }=153.06943363 \\
& \text { A15 }=127.96703226 \\
& \text { A17 }=90 \text {. } \\
& \text { A18 }=90 \text {. } \\
& \text { A19 }=104.52 \\
& \text { D1 }=-178.53818219 \\
& \text { D2 }=-133.87033211 \\
& \text { D3 }=\mathbf{9 . 5 3 4 2 5 8 2 8} \\
& \text { D4 }=\mathbf{5 4 . 7 6 6 2 0 0 6 2} \\
& \text { D5 }=174.31300511 \\
& \text { D6 }=-171.39120507 \\
& \text { D7 }=-5.75806162 \\
& \text { D8 }=179.52131799 \\
& \text { D9 }=179.29204232 \\
& \text { D10 }=-23.05714726 \\
& \text { D11 }=97.90186 \\
& \text { D12=-143.40314511 } \\
& \text { D13 }=8.30144484 \\
& \text { D14 }=-180 \text {. } \\
& \text { D15 }=0 \text {. } \\
& \text { D16 }=180 \text {. } \\
& \text { D17 }=-180 \text {. } \\
& \text { D18 }=\text {. }
\end{aligned}
$$

Complex 5:

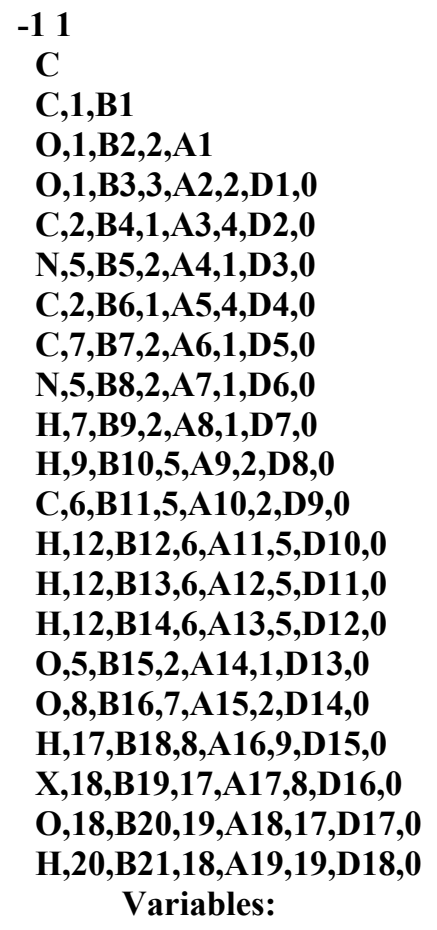


B18 $=1.79420004$

A16=119.17959704

Constants:

$B 1=1.55178421$

$B 2=1.24182114$

$B 3=1.23535246$

B4 $=\mathbf{2 . 4 0 9 0 6 6 7 2}$

$B 5=1.37732874$

B6 $=\mathbf{1 . 3 5 1 7 6 7 7 7}$

$B 7=1.43473251$

B8 $=1.37428629$

B9 $=1.07723985$

$B 10=1.00833225$

$B 11=1.46281973$

$B 12=1.08465789$

$B 13=1.08670697$

B14 $=1.08529274$

$B 15=1.21997852$

$B 16=1.22071407$

B19=1.

$\mathrm{B20}=\mathbf{0 . 9 5 7 2}$

$\mathrm{B} 21=0.9572$

A1 $=113.03957519$

A2 $=131.94153345$

A3 $=146.55041073$

A4 $=29.14472589$

A5 $=120.55297521$

A6 $=120.94699292$

A $7=\mathbf{8 5 . 4 8 9 7 0 9 1}$

$A 8=119.69565688$

A9 $=\mathbf{1 1 5 . 2 7 9 3 7 4 5 1}$

$A 10=117.04086245$

A11 $=107.66504377$

A12 $=\mathbf{1 1 0 . 3 9 7 3 0 0 6}$

A13=108.91093154

A14 $=153.06943363$

$A 15=127.96703226$

A17 $=90$.

A18 $=90$.

$\mathrm{A} 19=\mathbf{1 0 4 . 5 2}$

D1 $=-178.53818219$

D2 $=-133.87033211$

D3 $=9.53425828$

D4 $=\mathbf{5 4 . 7 6 6 2 0 0 6 2}$

D5 $=174.31300511$

D6 $=-\mathbf{1 7 1 . 3 9 1 2 0 5 0 7}$

D7 $=-5.75806162$

D8 $=179.52131799$

D9 $=179.29204232$

D10=-23.05714726

D11 $=97.90186$

D12 $=-143.40314511$

D13 $=8.30144484$

D14 $=-180$.

D15 $=0$.

D16 $=180$.

D17 $=180$.

D18 $=0$.

Complex 6: 


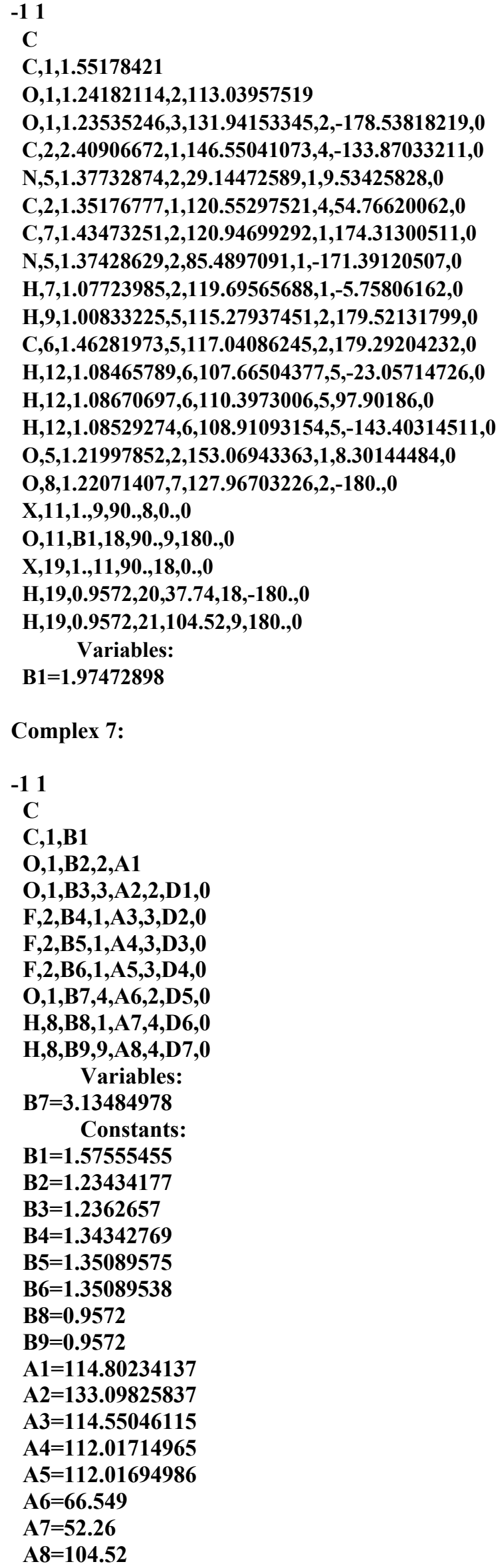




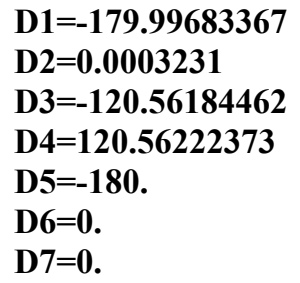

Complex 8:

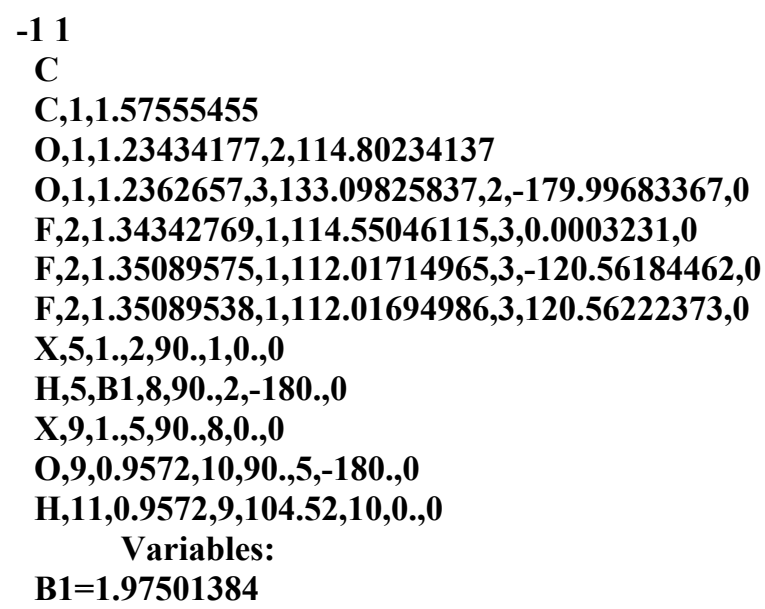

Complex 9:

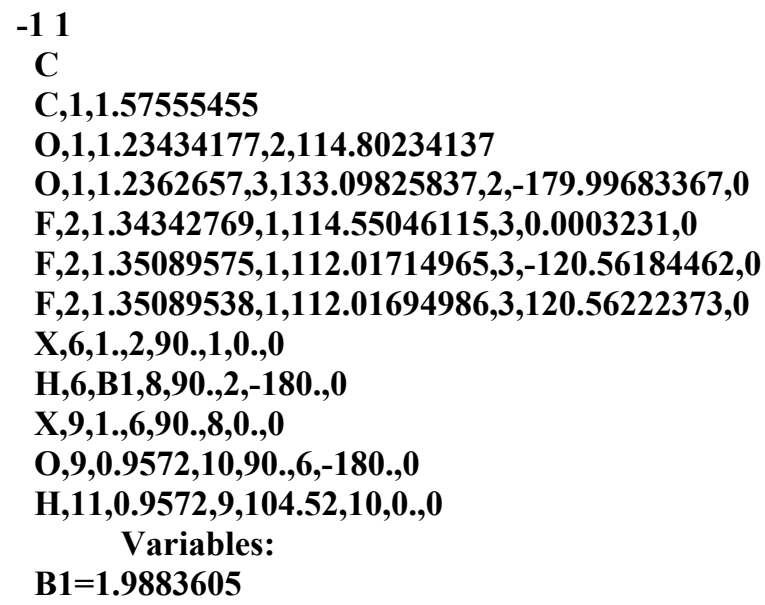

Complex 10:

$-11$

C

C,1,B1

$\mathrm{O}, 1, \mathrm{~B} 2,2, \mathrm{~A} 1$

$\mathrm{O}, 1, \mathrm{B3}, 3, \mathrm{~A} 2,2, \mathrm{D} 1,0$

$\mathrm{H}, 2, \mathrm{~B} 4,1, \mathrm{A3}, 3, \mathrm{D} 2,0$

$\mathrm{H}, 2, \mathrm{B5}, 1, \mathrm{~A} 4,3, \mathrm{D3}, 0$

$\mathrm{C}, 2, \mathrm{~B} 6,1, \mathrm{A5}, 3, \mathrm{D} 4,0$

N,7,B7,2,A6,1,D5,0

O,1,B8,3,A7,2,D6,0

H,9,B9, 1,A8,3,D7,0

H,9,B10,1,A9,4,D8,0

Variables: 


$$
\begin{aligned}
& \text { B8 }=3.13750451 \\
& \text { Constants: } \\
& B 1=1.5915448 \\
& B 2=1.23397194 \\
& B 3=1.24122709 \\
& B 4=1.08923954 \\
& B 5=1.08926316 \\
& B 6=1.45458947 \\
& B 7=1.14900709 \\
& B 9=0.96720003 \\
& B 10=0.96720023 \\
& A 1=116.60988448 \\
& A 2=131.94650414 \\
& \text { A3 }=107.40296811 \\
& \text { A4 }=107.37368399 \\
& \text { A5 }=116.49264135 \\
& \text { A6 }=177.93716009 \\
& A 7=65.97325 \\
& \text { A8 }=\mathbf{5 2 . 2 6} \\
& \text { A9 }=52.26 \\
& \text { D1 }=179.96284873 \\
& \text { D2 }=122.62669322 \\
& \text { D3 }=-122.23261896 \\
& \text { D4 }=\mathbf{0 . 1 8 0 1 7 6 3 4} \\
& \text { D5 }=180 \text {. } \\
& \text { D6 }=180 \text {. } \\
& \text { D7 }=\text {. } \text {. }
\end{aligned}
$$

Complex 11:

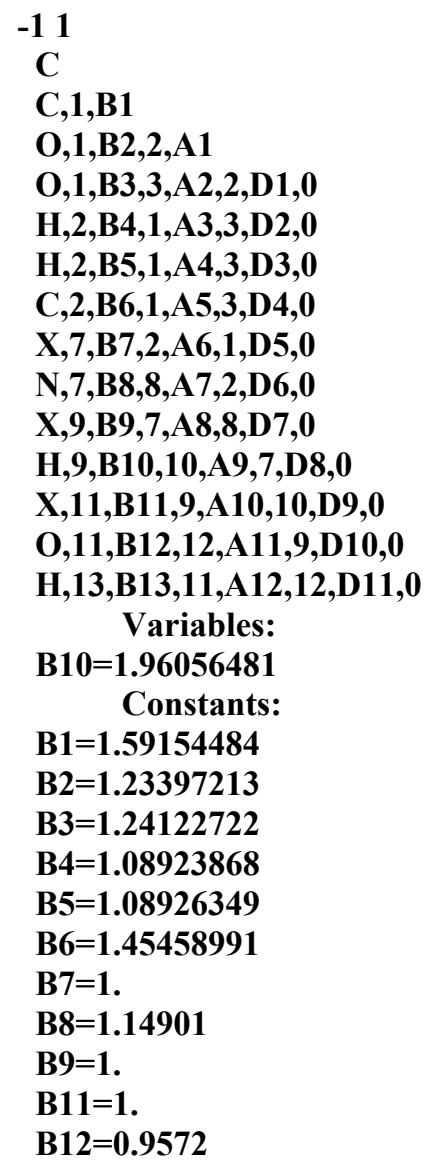




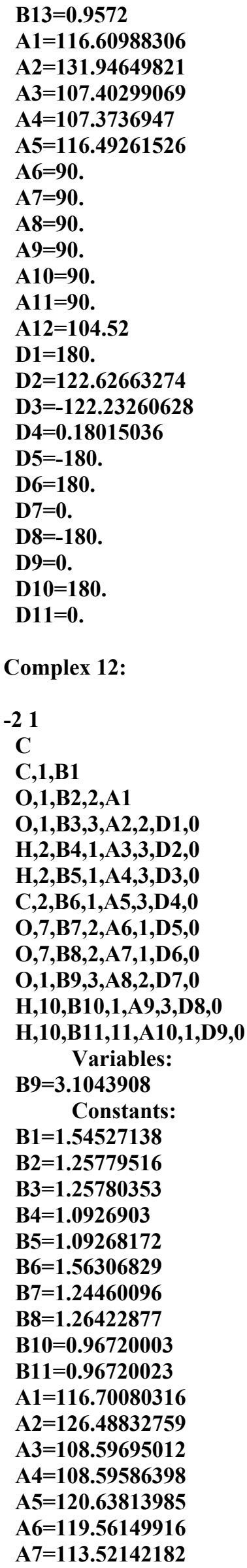




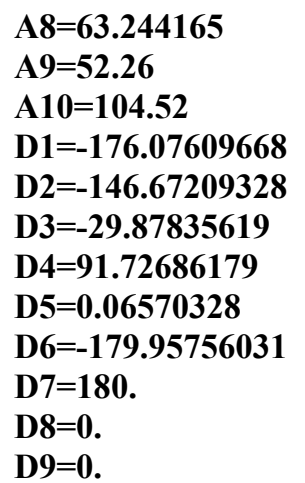

Complex 13:

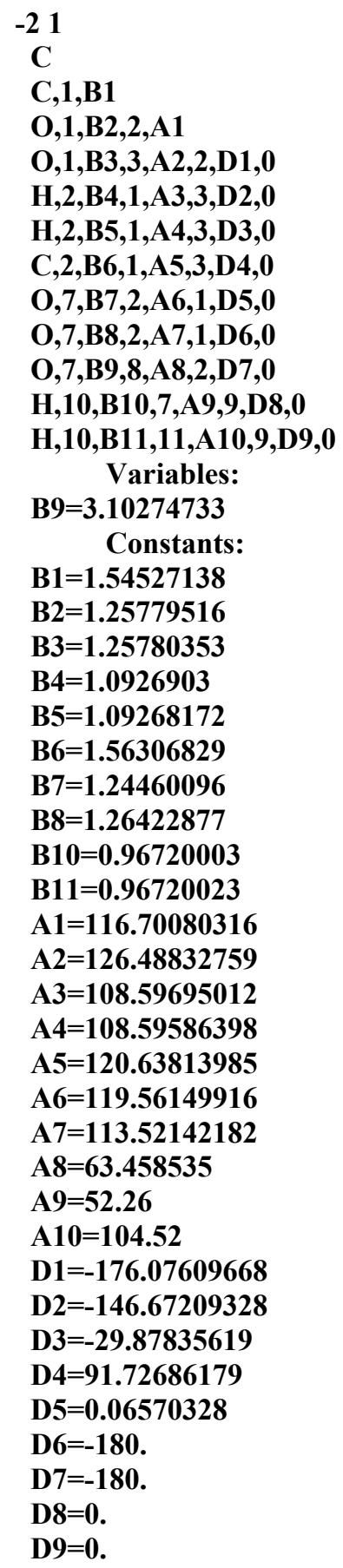


Complex 14:

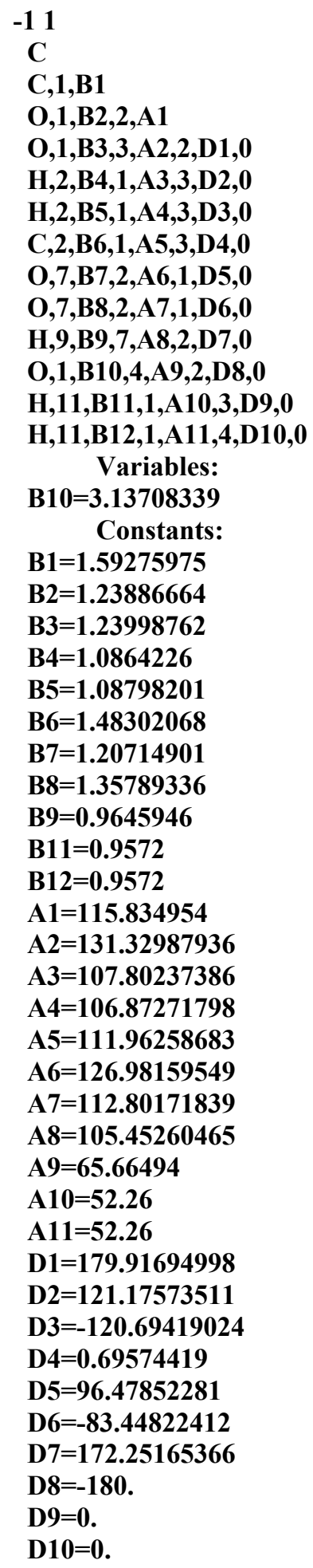

Complex 15:

$-11$

C

C,1,1.59275975

O,1,1.23886664,2,115.83495414

$0,1,1.23998762,3,131.3298794,2,179.91695004,0$ 
H,2,1.0864226,1,107.80237406,3,121.17573535,0

H,2,1.08798201,1,106.87271802,3,-120.69419047,0

C,2,1.48302068,1,111.9625867,3,0.69574412,0

O,7,1.20714901,2,126.98159541,1,96.47852341,0

0,7,1.35789336,2,112.8017184,1,-83.4482243,0

H,9,0.96459461,7,105.45260484,2,172.2516539,0

$\mathrm{H}, \mathbf{8 , B} 1,7, \mathrm{~A} 1,2,0 ., 0$

$\mathrm{X}, 11,1 ., 8,90 ., 7,180 ., 0$

O,11,0.9572,12,90.,8,180.,0

H,13,0.9572,11,104.52,12,0.,0 Variables:

B1 $=1.88181295$

A1 $=153.33741851$

Complex 16:

$-11$

C

C, $1,1.59275975$

O,1,1.23886664,2,115.83495414

$0,1,1.23998762,3,131.3298794,2,179.91695004,0$

H,2,1.0864226,1,107.80237406,3,121.17573535,0

H,2,1.08798201,1,106.87271802,3,-120.69419047,0

C,2,1.48302068,1,111.9625867,3,0.69574412,0

O,7,1.20714901,2,126.98159541,1,96.47852341,0

O,7,1.35789336,2,112.8017184,1,-83.4482243,0

H,9,0.96459461,7,105.45260484,2,172.2516539,0

$\mathrm{H}, 8, \mathrm{~B} 1,7, \mathrm{~A} 1,9,0 ., 0$

$\mathrm{X}, 11,1 ., 8,90 ., 7,180 ., 0$

$0,11,0.9572,12,90 ., 8,180 ., 0$

H,13,0.9572,11,104.52,12,0.,0 Variables:

$B 1=1.82613691$

A1 $=125.63136749$

Complex 17:

$-11$

C

C, $1,1.59275975$

O,1,1.23886664,2,115.83495414

O,1,1.23998762,3,131.3298794,2,179.91695004,0

H,2,1.0864226,1,107.80237406,3,121.17573535,0

H,2,1.08798201,1,106.87271802,3,-120.69419047,0

C,2,1.48302068,1,111.9625867,3,0.69574412,0

O,7,1.20714901,2,126.98159541,1,96.47852341,0

O,7,1.35789336,2,112.8017184,1,-83.4482243,0

H,9,0.96459461,7,105.45260484,2,172.2516539,0

$\mathrm{H}, 9, \mathrm{~B} 1,7, \mathrm{~A} 1,2,0 ., 0$

$\mathrm{X}, 11,1 ., 9,90 ., 7,0 ., 0$

O,11,0.9572,12,90.,9,-180.,0

H,13,0.9572,11,104.52,12,0.,0 Variables:

B1 $=2.01708378$

$\mathrm{A} 1=\mathbf{1 1 6 . 3 7 2 0 6 0 3 8}$

Complex 18:

$-11$

C 


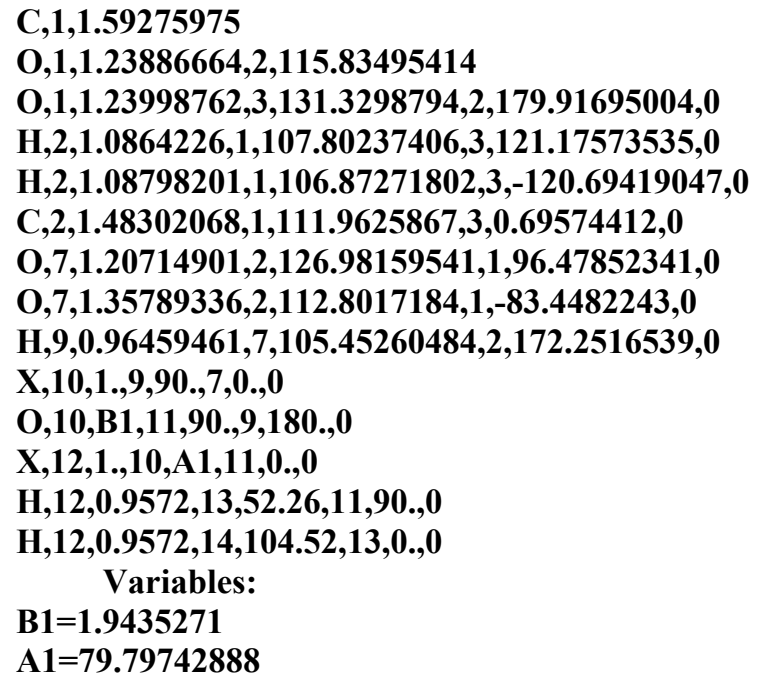

Complex 19:

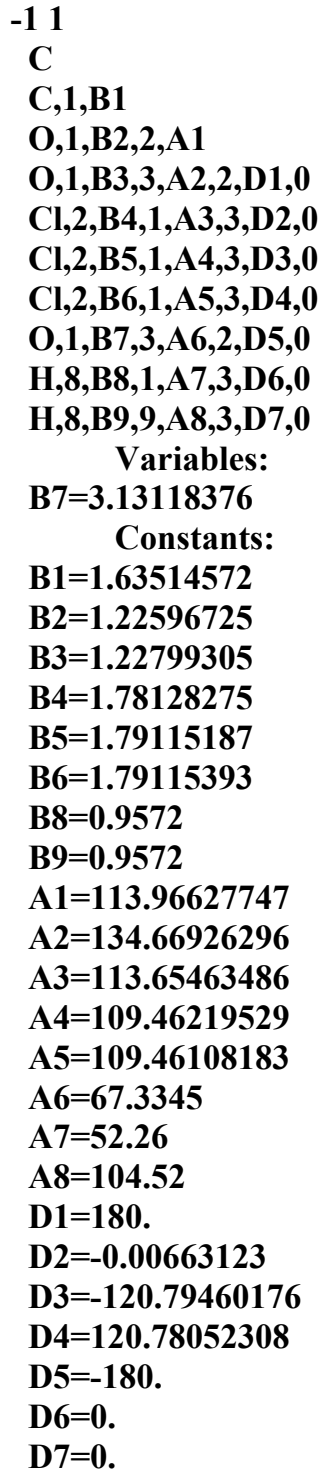

Complex 20: 
C

C,1,1.63514572

O,1,1.22596725,2,113.96627747

O,1,1.22799305,3,134.66926296,2,180.,0

Cl,2,1.78128275,1,113.65463486,3,-0.00663123,0

Cl,2,1.79115187,1,109.46219529,3,-120.79460176,0

Cl,2,1.79115393,1,109.46108183,3,120.78052308,0

$\mathrm{X}, 5,1 ., 2,90 ., 1,0 ., 0$

$\mathrm{H}, 5, \mathrm{~B} 1,8,90 ., 2,180 ., 0$

$\mathrm{X}, 9,1 ., 5,90 ., 8,0 ., 0$

$0,9,0.9572,10,90 ., 5,180 ., 0$

H,11,0.9572,9,104.52,10,0.,0 Variables:

B1 $=2.54414395$

Complex 21:

$-11$

C

C,1,1.63514572

O,1,1.22596725,2,113.96627747

O,1,1.22799305,3,134.66926296,2,180.,0

Cl,2,1.78128275,1,113.65463486,3,-0.00663123,0

Cl,2,1.79115187,1,109.46219529,3,-120.79460176,0

Cl,2,1.79115393,1,109.46108183,3,120.78052308,0

$\mathrm{X}, 7,1 ., 2,90 ., 1,0 ., 0$

$\mathrm{H}, 7, \mathrm{~B} 1,8,90 ., 2,180 ., 0$

$\mathrm{X}, 9,1 ., 7,90 ., 8,0 ., 0$

O,9,0.9572,10,90.,7,-180.,0

H,11,0.9572,9,104.52,10,0.,0

Variables:

B1 $=2.56426743$

Complex 22:

$-11$

C

O,1,B1

$\mathrm{O}, 1, \mathrm{~B} 2,2, \mathrm{~A} 1$

$\mathrm{C}, 1, \mathrm{B3}, 3, \mathrm{~A} 2,2, \mathrm{D} 1,0$

$\mathrm{H}, 4, \mathrm{~B} 4,1, \mathrm{~A} 3,3, \mathrm{D} 2,0$

H,4,B5,1,A4,3,D3,0

C,4,B6,1,A5,3,D4,0

C,7,B7,4,A6,1,D5,0

H,8,B8, 7,A7,4,D6,0

H,8,B9, 7,A8,4,D7,0

H,8,B10,7,A9,4,D8,0

O,7,B11,4,A10,1,D9,0

O,1,B12,2,A11,4,D10,0

H,13,B13,1,A12,2,D11,0

H,13,B14,14,A13,2,D12,0

Variables:

B12=3.14805818

Constants:

B1 $=1.24436989$

B2 $=1.23930451$

$B 3=1.58793488$

B4 $=\mathbf{1 . 0 9 3 1 9 9 2 8}$

$B 5=1.08685943$ 


$$
\begin{aligned}
& \text { B6 }=1.4959442 \\
& \text { B7 }=1.50925758 \\
& B 8=1.09053588 \\
& \text { B9=1.09224139 } \\
& B 10=1.08749615 \\
& \text { B11 }=1.21545785 \\
& B 13=0.96720003 \\
& \text { B14 }=0.96720023 \\
& \text { A1 }=130.21217884 \\
& \text { A2 }=113.22750517 \\
& \text { A3 }=\mathbf{1 0 4 . 8 6 8 2 1 9 2 5} \\
& \text { A4 }=108.28486641 \\
& \text { A5 }=115.19767649 \\
& A 6=116.56311525 \\
& \text { A } 7=107.11293125 \\
& \text { A8 }=109.64052644 \\
& \text { A9=110.1801297 } \\
& \mathrm{A} 10=122.55256577 \\
& A 11=65.10609 \\
& \mathrm{~A} 12=\mathbf{5 2 . 2 6} \\
& \text { A13 }=104.52 \\
& \text { D1 }=178.10252484 \\
& \text { D2 }=-73.64554395 \\
& \text { D3 }=42.47375656 \\
& \text { D4 }=165.86009468 \\
& \text { D5 }=68.2212359 \\
& \text { D6 }=-57.62801748 \\
& \text { D7 }=\mathbf{5 9 . 1 9 1 7 7 4} \\
& \text { D8 }=-\mathbf{1 7 9 . 4 1 4 1 4 7 4 3} \\
& \text { D9=-111.55050305 } \\
& \text { D10 }=180 \text {. } \\
& \text { D11 }=\text {. } \\
& \text { D12 }=\text { 0. }
\end{aligned}
$$

Complex 23:

$-11$

C

$$
\text { O,1,1.24436989 }
$$

O,1,1.23930451,2,130.21217884

C,1,1.58793488,3,113.22750517,2,178.10252484,0

H,4,1.09319928,1,104.86821925,3,-73.64554395,0

H,4,1.08685943,1,108.28486641,3,42.47375656,0

C,4,1.4959442,1,115.19767649,3,165.86009468,0

C,7,1.50925758,4,116.56311525,1,68.2212359,0

H,8,1.09053588,7,107.11293125,4,-57.62801748,0

H,8,1.09224139,7,109.64052644,4,59.191774,0

H,8,1.08749615,7,110.1801297,4,-179.41414743,0

O,7,1.21545785,4,122.55256577,1,-111.55050305,0

$\mathrm{H}, 12, \mathrm{~B} 1,7, \mathrm{~A} 1,8,0 ., 0$

$\mathrm{X}, 13,1 ., 12,90 ., 7,180 ., 0$

$0,13,0.96720023,14,90 ., 12,180 ., 0$

H,15,0.96720023,13,104.52,14,0.,0 Variables:

B1 $=1.81661254$

A1 $=123.56531314$

Complex 24:

$-11$ 


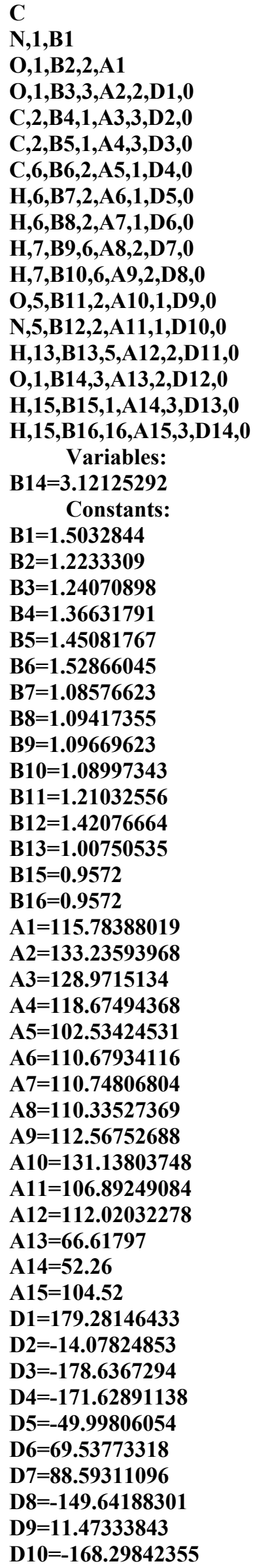




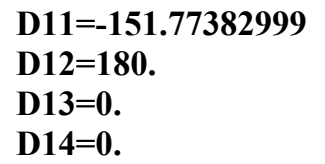

Complex 25:

$-11$

C

N,1,B1

$\mathrm{O}, 1, \mathrm{~B} 2,2, \mathrm{~A} 1$

$\mathrm{O}, 1, \mathrm{B3}, 3, \mathrm{~A} 2,2, \mathrm{D} 1,0$

$\mathrm{C}, 2, \mathrm{~B} 4,1, \mathrm{A3}, 3, \mathrm{D} 2,0$

$\mathrm{C}, 2, \mathrm{B5}, 1, \mathrm{~A} 4,3, \mathrm{D3}, 0$

C,6,B6,2,A5,1,D4,0

H,6,B 7,2,A6,1,D5,0

H,6,B8,2,A7,1,D6,0

H,7,B9,6,A8,2,D7,0

H,7,B10,6,A9,2,D8,0

O,5,B11,2,A10,1,D9,0

N,5,B12,2,A11,1,D10,0

H,13,B13,5,A12,2,D11,0

$\mathrm{X}, 12, \mathrm{~B} 14,5, \mathrm{A13}, 13, \mathrm{D} 12,0$

H,12,B15,15,A14,5,D13,0

$\mathrm{X}, 16, \mathrm{~B} 16,12, \mathrm{A15}, 15, \mathrm{D} 14,0$

$\mathrm{O}, 16, \mathrm{~B} 17,17, \mathrm{~A} 16,2, \mathrm{D} 15,0$

H,18,B18,16,A17,17,D16,0

Variables:

B15=1.85872987

Constants:

B1 $=1.5032844$

B2 $=1.2233309$

$B 3=1.24070898$

B4 $=1.36631791$

$B 5=1.45081767$

B6 $=1.52866045$

$B 7=1.08576623$

B8 $=1.09417355$

B9 $=1.09669623$

$B 10=1.08997343$

$B 11=1.21032556$

$B 12=1.42076664$

B13 $=1.00750535$

B14 $=1$.

$\mathrm{B} 16=1$.

$\mathrm{B} 17=0.9572$

$\mathrm{B} 18=0.9572$

A1 $=\mathbf{1 1 5 . 7 8 3 8 8 0 1 9}$

A2 $=133.23593968$

A3 $=128.9715134$

A4 $=118.67494368$

A5 $=102.53424531$

A6 $=110.67934116$

A $7=110.74806804$

A8 $=110.33527369$

A9 $=112.56752688$

$\mathrm{A10}=\mathbf{1 3 1 . 1 3 8 0 3 7 4 8}$

$A 11=106.89249084$

$A 12=112.02032278$

A13 $=$ 90. 


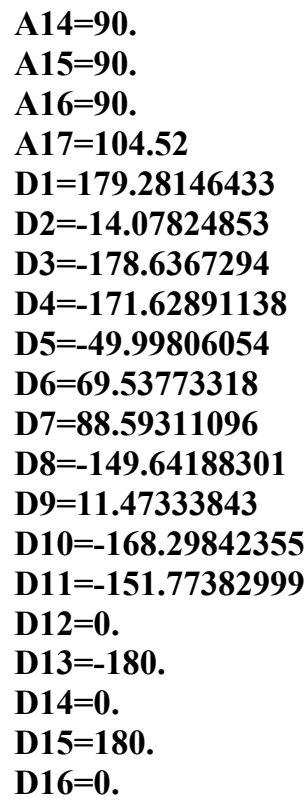

Complex 26:

$-11$

C

N,1,1.5032844

O,1,1.2233309,2,115.78388019

O,1,1.24070898,3,133.23593968,2,179.28146433,0

C,2,1.36631791,1,128.9715134,3,-14.07824853,0

C,2,1.45081767,1,118.67494368,3,-178.6367294,0

C,6,1.52866045,2,102.53424531,1,-171.62891138,0

H,6,1.08576623,2,110.67934116,1,-49.99806054,0

H,6,1.09417355,2,110.74806804,1,69.53773318,0

H,7,1.09669623,6,110.33527369,2,88.59311096,0

H,7,1.08997343,6,112.56752688,2,-149.64188301,0

0,5,1.21032556,2,131.13803748,1,11.47333843,0

$\mathrm{N}, 5,1.42076664,2,106.89249084,1,-168.29842355,0$

H,13,1.00750535,5,112.02032278,2,-151.77382999,0

$\mathrm{X}, 14,1 ., 13,90 ., 5,0 ., 0$

O,14,B1,15,90.,13,-180.,0

$\mathrm{X}, 16,1 ., 14,90 ., 15,0 ., 0$

H,16,0.9572,17,90.,15,127.74,0

H,16,0.9572,18,104.52,17,-90.,0 Variables:

$B 1=2.21925021$

Complex 27:
$-11$
C
$\mathrm{C}, 1, \mathrm{~B} 1$
$\mathrm{O}, 1, \mathrm{~B} 2,2, \mathrm{~A} 1$
O,1,B3,3,A2,2,D1,0
$\mathrm{H}, 2, \mathrm{~B} 4,1, \mathrm{~A} 3,3, \mathrm{D} 2,0$
$\mathrm{H}, 2, \mathrm{B5}, 1, \mathrm{~A} 4,3, \mathrm{D3}, \mathrm{O}$
N,2,B6,1,A5,3,D4,0
O,7,B7,2,A6,1,D5,0
$\mathrm{O}, 7, \mathrm{B8}, 2, \mathrm{~A} 7,1, \mathrm{D6}, 0$
O,1,B9,3,A8,2,D7,0
H,10,B10,1,A9, 7,D8,0 


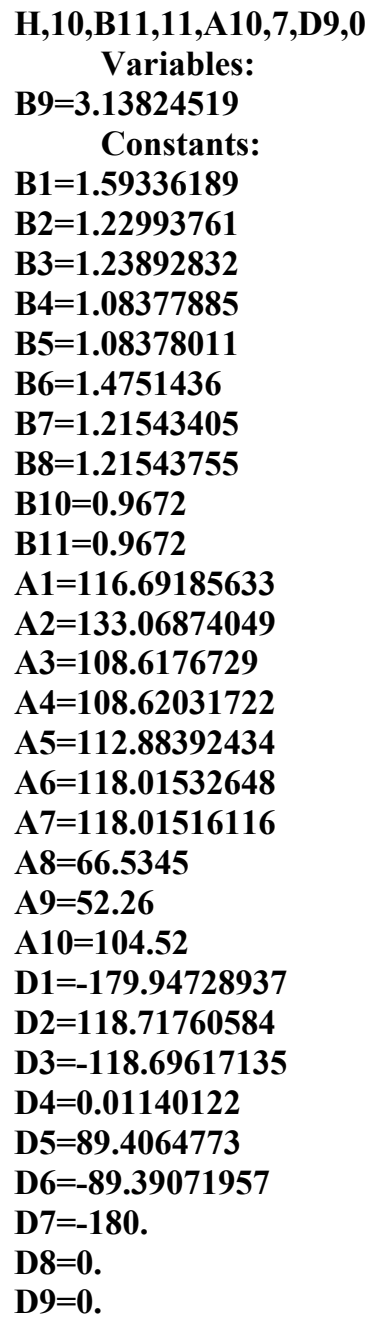

Complex 28:

$-11$

C

C,1,1.59336189

O,1,1.22993761,2,116.69185633

O,1,1.23892832,3,133.06874049,2,-179.94728937,0

H,2,1.08377885,1,108.6176729,3,118.71760584,0

H,2,1.08378011,1,108.62031722,3,-118.69617135,0

$\mathrm{N}, 2,1.4751436,1,112.88392434,3,0.01140122,0$

O,7,1.21543405,2,118.01532648,1,89.4064773,0

O,7,1.21543755,2,118.01516116,1,-89.39071957,0

O,7,B1,8,61.979,2,180.,0

$\mathrm{H}, 10,0.9572,7,52.26,8,0 ., 0$

H,10,0.9672,11,104.52,9,0.,0

Variables:

B1=3.36141079

Complex 29:

$$
-11
$$

C

C

C

H

$\begin{array}{llll}\mathbf{1} & \text { B1 } & & \\ \mathbf{1} & \text { B2 } & \mathbf{2} & \text { A1 } \\ \mathbf{2} & \text { B3 } & \text { 1 } & \text { A2 } \\ 2 & \text { B4 } & \text { 1 } & \text { A3 }\end{array}$




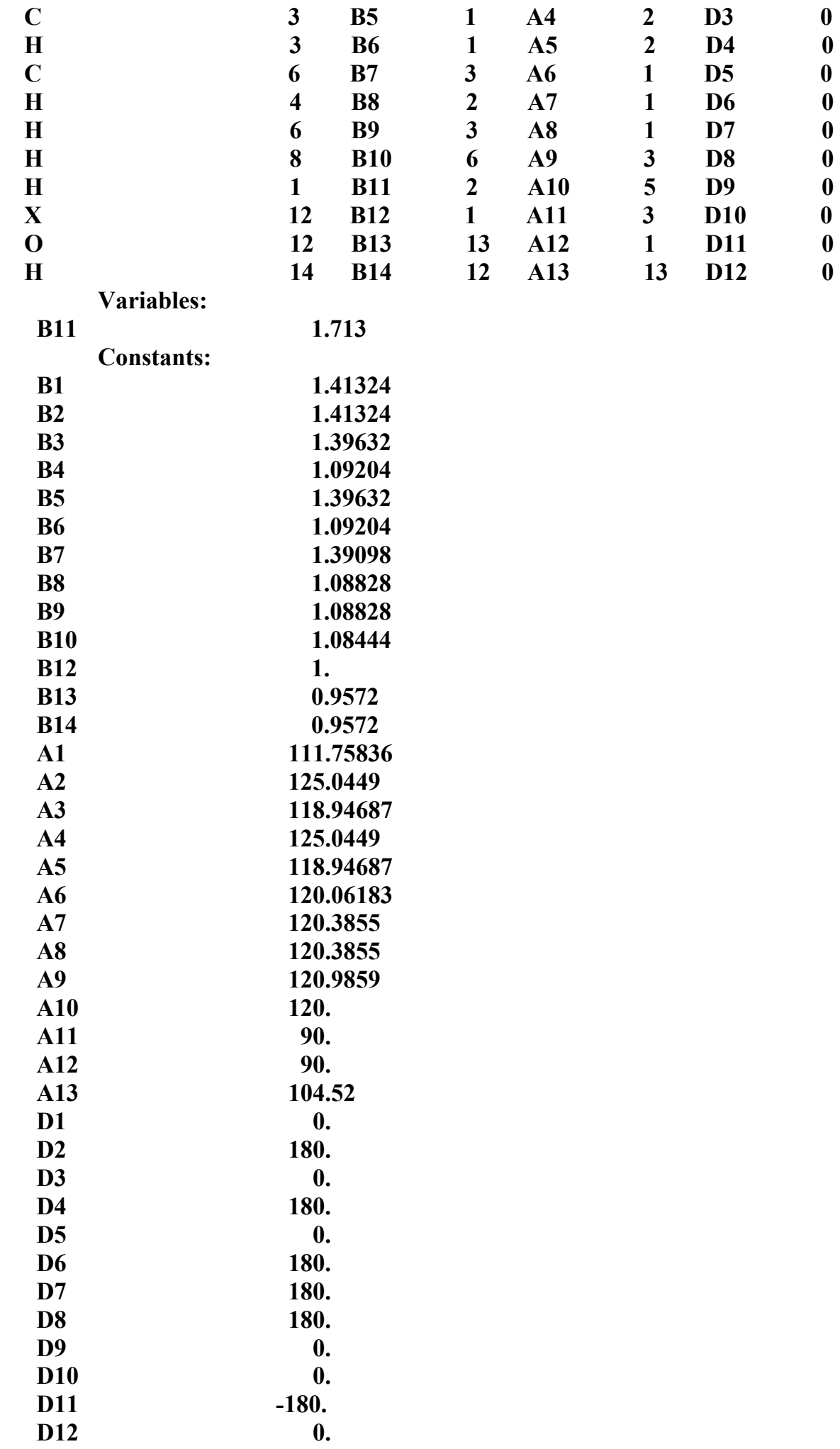

Complex 30:

$$
\begin{gathered}
-11 \\
\mathbf{C} \\
\mathbf{C} \\
\mathbf{N} \\
\mathrm{C}
\end{gathered}
$$$$
\text { B1 }
$$$$
\begin{array}{lll}
\text { B2 } & 1 & \text { A1 }
\end{array}
$$$$
\text { B3 } 3
$$$$
\text { A2 } 2
$$$$
\text { D1 } 0
$$ 


\begin{tabular}{|c|c|c|c|c|c|c|}
\hline C & 4 & B4 & 1 & $\mathbf{A 3}$ & 3 & D2 \\
\hline $\mathbf{N}$ & 2 & B5 & 1 & A4 & 4 & D3 \\
\hline H & 4 & B6 & 1 & A5 & 3 & D4 \\
\hline H & 6 & B7 & 2 & A6 & 1 & D5 \\
\hline C & 3 & B8 & 2 & A7 & 1 & D6 \\
\hline H & 9 & B9 & 3 & A8 & 2 & D7 \\
\hline H & 9 & B10 & 3 & A9 & 2 & D8 \\
\hline H & 9 & B11 & 3 & $\mathbf{A 1 0}$ & 2 & D9 \\
\hline O & 2 & B12 & 1 & A11 & 4 & D10 \\
\hline O & 5 & B13 & 4 & A12 & 1 & D11 \\
\hline H & 1 & B14 & 4 & A13 & 7 & D12 \\
\hline $\mathbf{X}$ & 15 & B15 & 1 & A14 & 4 & D13 \\
\hline O & 15 & B16 & 16 & A15 & 1 & D14 \\
\hline H & 17 & B17 & 15 & A16 & 16 & D15 \\
\hline
\end{tabular}

variables:

B14

1.812

A13 $\quad 127.5$

constants:

B1

2.47312297

1.37122046

B3

1.37584031

1.43010606

1.36989500

1.08205415

1.00768644

$\mathbf{1 . 4 5 3 6 7 0 5 4}$

1.09147720

$\begin{array}{ll}\text { B10 } & \mathbf{1 . 0 9 1 4 7 8 5 9} \\ \text { B11 } & \mathbf{1 . 0 8 4 4 4 0 5 9}\end{array}$

B12 1.22987531

B13 $\quad 1.23137351$

B15 1.0

B16 0.95720000

B17 $\quad \mathbf{0 . 9 5 7 2 0 0 0 0}$

A1 $\quad \mathbf{2 7 . 1 7 9 1 5 3 6 0}$

A2 $\quad 114.78379277$

A3 $\quad 124.77121594$

A4 $\quad \mathbf{8 7 . 1 3 4 5 5 2 6 2}$

A5 $\quad 119.87298053$

A6 $\quad 116.08257753$

A7 $\quad \mathbf{1 1 4 . 5 5 4 2 5 4 0 7}$

A8 $\quad 110.88698934$

A9 $\quad \mathbf{1 1 0 . 8 8 7 1 6 2 0 2}$

A10 $\quad 106.36418628$

A11 $\quad 151.12509040$

A12 129.10010427

A14 90.0

$\mathbf{A 1 5} \quad \mathbf{8 0 . 6 9 6 7 8 3 9 0}$

A16 $\quad 104.52$

D1 0.00035403

D2 $\quad-\mathbf{0 . 0 0 0 2 7 4 6 1}$

0.00000000

179.99970903

$-180.00000000$

$-179.99946716$

$-\mathbf{5 9 . 6 7 2 7 0 6 2 8}$

$\begin{array}{lr}\text { D8 } & \mathbf{5 9 . 6 7 8 6 9 7 1 8} \\ \text { D9 } & -\mathbf{1 7 9 . 9 9 7 2 3 8 6 7}\end{array}$

D10 179.99958346

D11 179.99952011 


$\begin{array}{ll}\text { D12 } & \text { 0.0 } \\ \text { D13 } & \text { 90.0 } \\ \text { D14 } & -180.0 \\ \text { D15 } & 0.0\end{array}$

Complex 31

-1
C
F
F
F
H
X

variables:

B4 $\quad 1.887$

constants:

B1

B2

B3

B5

B6

B7

A1

A2

A3

A4

A5

A6

D1

D2

D3

D4

D5

Complex 32:

-11
C
H
H
C
N
H
X
O
H

variables:

constants:

$\begin{array}{lr}\text { B1 } & \mathbf{1 . 0 7 9 6 3 4 7 6} \\ \text { B2 } & \mathbf{1 . 0 7 9 6 3 4 7 6} \\ \text { B3 } & \mathbf{1 . 3 8 2 5 8 2 1 8} \\ \text { B4 } & \mathbf{1 . 1 7 3 5 1 7 7 9} \\ \text { B6 } & \mathbf{1 . 0 0} \\ \text { B7 } & \mathbf{0 . 9 5 7 2 0 0 0 0} \\ \text { B8 } & \mathbf{0 . 9 5 7 2 0 0 0 0} \\ \text { A1 } & 118.33039609\end{array}$

1.41799006

1.41799085

1.41799085

1.0

0.95720000

0.95720000

$\mathbf{9 9 . 7 2 0 2 9 1 1 4}$

99.72029114

114.55

90.0

90.0

104.52

101.72038245

$-123.92$

$-180.0$

$-180.0$

0.0
B5

$\begin{array}{ll}\text { H } & 1 \\ \text { H } & 1 \\ \text { C } & 1 \\ \text { N } & 4 \\ \text { H } & 1 \\ \text { X } & 6 \\ \text { H } & 6 \\ \text { H } & 8\end{array}$

A1

1
1
1
4
1
6
6
8

1.717
118.33039609

$\begin{array}{llllll}\text { B1 } & & & & & \\ \text { B2 } & 2 & \text { A1 } & & \text { D1 } & \text { 0 } \\ \text { B3 } & 2 & \text { A2 } & 3 & \text { D2 } & \text { 0 } \\ \text { B4 } & 2 & \text { A3 } & 3 & \text { D3 } & \text { 0 } \\ \text { B5 } & 1 & \text { A4 } & 2 & \text { D4 } & \text { 0 } \\ \text { B6 } & 6 & \text { A5 } & 1 & \text { D5 } & \text { 0 }\end{array}$

$\begin{array}{ll}\text { B1 } & \\ \text { B2 } & 2 \\ \text { B3 } & 2 \\ \text { B4 } & 1 \\ \text { B5 } & 4 \\ \text { B6 } & 1 \\ \text { B7 } & 7 \\ \text { B8 } & 6\end{array}$

A1

A2 3

A3 2

A4 5

A5 4

A6 1

A7 7

$\begin{array}{ll}\text { D1 } & \text { 0 } \\ \text { D2 } & \text { 0 } \\ \text { D3 } & \text { 0 } \\ \text { D4 } & \text { 0 } \\ \text { D5 } & \text { 0 } \\ \text { D6 } & \text { 0 }\end{array}$ 


$\begin{array}{lc}\text { A2 } & \mathbf{1 1 8 . 7 8 2 6 4 3 3 9} \\ \text { A3 } & \mathbf{1 7 8 . 3 1 8 3 4 6 6 9} \\ \text { A4 } & 90.0 \\ \text { A5 } & 90.00 \\ \text { A6 } & 90.0 \\ \text { A7 } & \mathbf{1 0 4 . 5 2} \\ \text { D1 } & -156.96836274 \\ \text { D2 } & -101.56826978 \\ \text { D3 } & 0.0 \\ \text { D4 } & -180.0 \\ \text { D5 } & -180.0 \\ \text { D6 } & 0.00\end{array}$

Complex 33:

C

C

H

$\begin{array}{ll}\text { H } & 1 \\ \text { C } & 1\end{array}$

O 4

O 4

H 6

H 1

X 8

$\begin{array}{rr}\text { O } & 8 \\ \text { H } & 10\end{array}$

variables:

B7

constants:

2.073

B1

$\mathbf{1 . 0 7 8 3 4 5 4 6}$

B2 $\quad \mathbf{1 . 0 7 8 5 1 9 5 2}$

B3 $\quad 1.37309138$

B4 $\quad 1.25256638$

B5 $\quad \mathbf{1 . 4 1 9 2 1 9 0 6}$

B6 0.96204249

B8 $\quad 1.00$

B9 $\quad \mathbf{0 . 9 5 7 2 0 0 0 0}$

B10 $\quad 0.95720000$

A1 $\quad \mathbf{1 2 0 . 3 1 4 5 7 0 0 3}$

A2 $\quad 119.07127670$

A3 131.23049741

A4 114.42195143

A5 $\quad 101.23714272$

A6 $\quad 90.0$

A7 $\quad 90.0$

A8 $\quad 90.0$

A9 $\quad 104.52$

D1 $\quad-179.94607651$

D2 $\quad \mathbf{- 0 . 0 4 9 0 9 1 1 1}$

D3 $\quad-179.98493730$

D4 179.95794451

D5 90.0

D6 $\quad-180.0$

D7 $\quad-\mathbf{1 8 0 . 0}$

D8 0.0

Complex 34:

$-11$ 


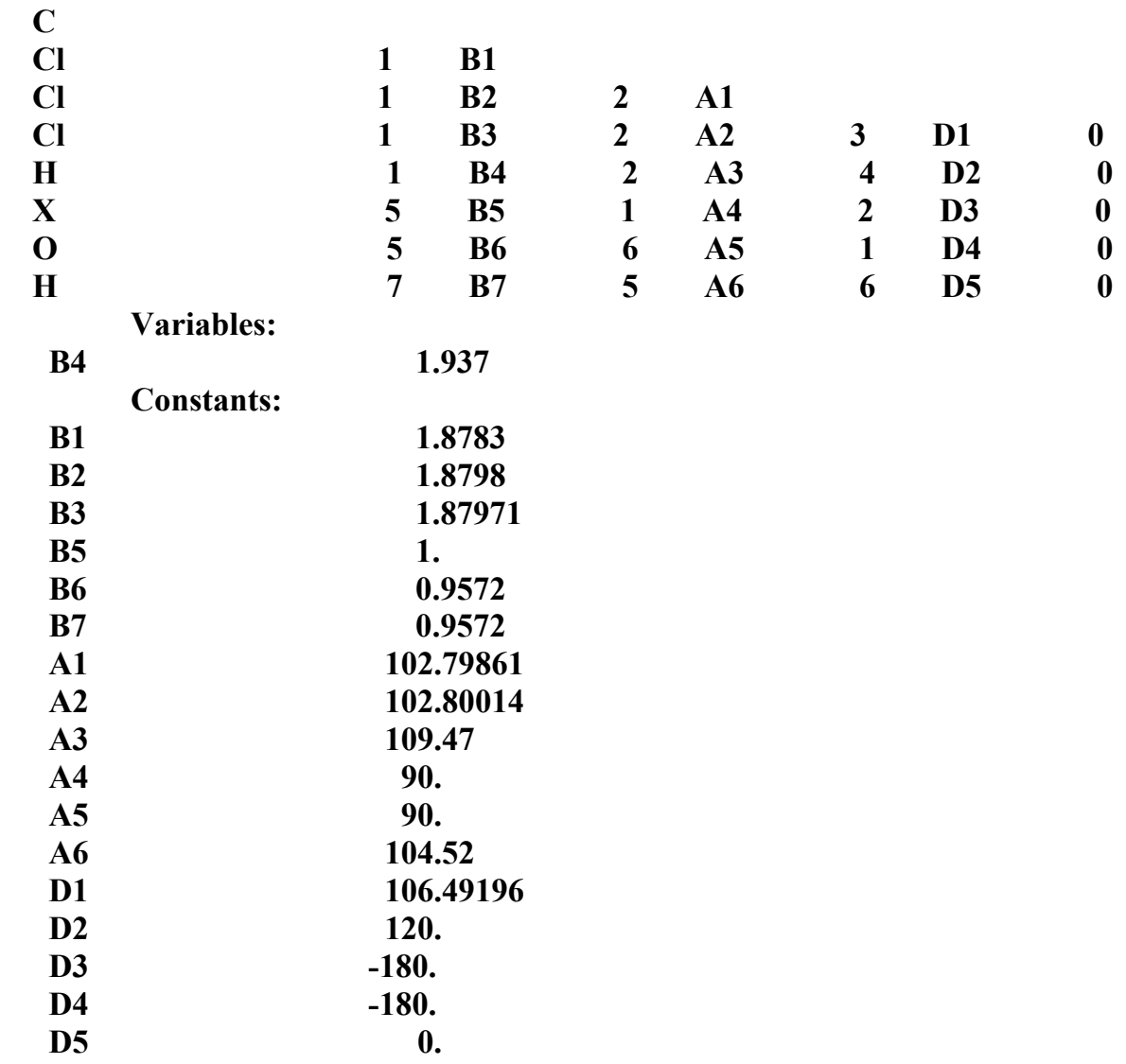

